\title{
CURVATURE AND FUNCTION THEORY ON RIEMANNIAN MANIFOLDS
}

\author{
PETER LI
}

Function theory on Euclidean domains in relation to potential theory, partial differential equations, probability, and harmonic analysis has been the target of investigation for decades. There is a wealth of classical literature in the subject. Geometers began to study function theory with the primary reason to prove a uniformization type theorem in higher dimensions. It was first proposed by Greene-Wu and Yau to study the existence of bounded harmonic functions on a complete manifold with negative curvature. While uniformization in dimension greater than 2 still remains an open problem, the subject of function theory on complete manifolds takes on life of its own. The seminal work of Yau [107] provided a fundamental technique in handling analysis on noncompact, complete manifolds. It also opens up many interesting problems which are essential for the understanding of analysis on complete manifolds. Since Yau's paper in 1975, there are many developments in this subject. The aim of this article is to give a rough outline of the history of a specific point of view in this area, namely, the interplay between the geometry primarily the curvature - and the function theory. Throughout this article, unless otherwise stated, we will assume that $M^{n}$ is an $n$-dimensional, complete, non-compact, Riemannian manifold without boundary. In this case, we will simply say that $M$ is a complete manifold.

One of the goal of this survey is to demonstrate, by way of known theorems, the two major steps which are common in many geometric analysis programs. First, we will show how one can use assumptions on the curvature to conclude function theoretic properties of the manifold $M$. Secondly, we will showed that function theoretic properties can in turn be used to conclude geometrical and topological statements about

Research partially supported by NSF grant \#DMS-9626310. 
the manifold. In many incidents, combining the two steps will result in a theorem which hypothesizes on the curvature and concludes on either the topological, geometrical, or complex structure of the manifold.

The references will not be comprehensive due to the vast literature in the subject. It is merely an indication of the flavor of the field for the purpose of whetting one's appetite. As examples of areas not being discussed in this note are harmonic analysis (function theory) on symmetric spaces, Lie groups, and discrete groups. The contributors to this subject are Furstenberg, Varopoulos, Coulhon, Saloff-Coste, and etc. Another point of view which was systematically taken up by Lyons-Sullivan, and later by Varopoulos, is to relate the group theoretic property of the covering group to the function theory of a covering space.

\section{Curvature assumptions and notations}

In this paper, we will impose different curvature assumptions on various occasions. The two primary notions of curvature we will use are the sectional curvature and the Ricci curvature. For a given point $x \in M$ and a 2-plane section $\sigma \subset T_{x} M$, we denote its sectional curvature by $K_{M}(\sigma)$. The notation $K_{M}(x)$ means the sectional curvature functional defined on all 2-plane sections at the point $x$. The Ricci curvature will be denoted by $\operatorname{Ric}_{M}(x)$, which is a symmetric 2-tensor at the point $x \in M$. In the first half of this paper, there are primarily four different types of curvature assumptions that are related to one another.

(1) Non-negative Ricci curvature: We assume that $M$ has non-negative Ricci curvature at every point, i.e.,

$$
\operatorname{Ric}_{M}(x) \geq 0
$$

for all $x \in M$.

(2) Non-negative Ricci curvature near infinity: There exists a compact subset $D \subset M$, such that

$$
\operatorname{Ric}_{M}(x) \geq 0
$$

for all $x \in M \backslash D$.

(3) Asymptotically non-negative Ricci curvature: There exists a monotonically non-increasing function $\alpha(r)>0$ satisfying

$$
\int_{0}^{\infty} r^{n-1} \alpha(r) d r<\infty,
$$

such that,

$$
\operatorname{Ric}_{M}(x) \geq-\alpha(\rho(x)),
$$


where $\rho(x)$ is the distance function from a fixed point $p \in M$.

(4) Almost non-negative Ricci curvature: There exists a sufficiently small $\epsilon>0$, such that,

$$
\operatorname{Ric}_{M}(x) \geq-\epsilon \rho^{-2}(x)
$$

for all $x \in M$.

One easily verifies that the above assumptions satisfy the following monotonically decreasing ordering:

$$
(1) \Rightarrow(2) \Rightarrow(3) \Rightarrow(4) \text {. }
$$

We would also like to take this opportunity to point out that assumptions on the Ricci curvature yield much less information on the manifold as similar assumptions on the sectional curvature. For instance, the soul theorem of Cheeger-Gromoll asserts that:

Theorem 1.1 (Cheeger-Gromoll [22]). If $M$ has non-negative sectional curvature, then there exists a compact totally geodesic submanifold $N \subset M$ such that $M$ is diffeomorphic to the normal bundle of $N$.

The sectional curvature assumption places stringent topological restriction on a manifold. In particular, $M$ must have the topological type of a compact manifold. In a similar spirit, Abresch took their argument a step further.

Theorem 1.2 (Abresch [1], [2]). Suppose $M$ has asymptotically nonnegative sectional curvature, i.e., there exists a positive, monotonically non-increasing function $\gamma(r)$ satisfying

$$
\int_{0}^{\infty} r \gamma(r) d r<\infty,
$$

such that, $K_{M}(x) \geq-\gamma(\rho(x))$ for all $x \in M$. Then $M$ must have bounded topological type. Moreover, the number of ends of $M$ and the total Betti number of $M$ can be estimated in terms of $n$ and $\gamma$.

Contrary to the rigid topological restriction imposed on a manifold with the sectional curvature assumptions in the last two theorems, ShaYang [97] showed that there are manifolds with positive Ricci curvature which has infinite topological type. In fact, their example is diffeomorphic to $\mathbb{R}^{4}$ connected sum with $k$ copies of $\mathbb{C P}^{2}$, for any $k=1,2, \ldots, \infty$. Notice that the notions of asymptotically non-negative sectional curvature and asymptotically non-negative Ricci curvature differs by a factor of $r^{n-1}$ in the integrand. This factor seems to arise more naturally for 
Ricci curvature than sectional curvature. However, there are no concrete examples which indicate that this factor is not a mere technical assumption.

Definition 1.3. Let $D \subset M$ be a compact subset of $M$. An end $E$ of $M$ with respect to $D$ is a connected unbounded component of $M \backslash D$. When we say that $E$ is an end, it is implicitly assumed that $E$ is an end with respect to some compact subset $D \subset M$.

From the definition, it is clear that if $D_{1}$ and $D_{2}$ are compact subsets with $D_{1} \subset D_{2}$, then the number of ends with respect to $D_{1}$ is at most the number of ends with respect to $D_{2}$. This monotonicity property allows us to define the number of ends of a manifold.

Definition 1.4. $M$ is said to have finitely many ends if there exists $0<k<\infty$, such that, for any $D \subset M$, the number of ends with respect to $D$ is at most $k$.

In this case, we denote $\pi_{0}^{\infty}(M)$ to be the smallest such $k$. Obviously, $\pi_{0}^{\infty}(M)$ must be an integer. Also, one readily concludes that there exists $D_{0} \subset M$, such that, the number of ends with respect to $D_{0}$ is precisely $\pi_{0}^{\infty}(M)$. If $M$ has infinitely ends, we will still use $\pi_{0}^{\infty}(M)=\infty$ to denote the number of ends.

\section{Function theory}

Definition 2.1. A Green's function $G(x, y)$ is a function defined on $(M \times M) \backslash\{(x, x)\}$ satisfying the following properties:

- $G(x, y)=G(y, x)$, and

- $\Delta_{y} G(x, y)=-\delta_{x}(y)$,

for all $x \neq y$.

It was proved by Malgrange [84] that every manifold admits a Green's function. Recently, Li-Tam [69] gave a constructive argument for the existence of $G(x, y)$. As in the difference between $\mathbb{R}^{2}$ and $\mathbb{R}^{n}$ for $n \geq 3$, some manifolds admit Green's functions which are positive and others may not. This special property distinguishes the function theory of complete manifolds into two classes.

Definition 2.2. A complete manifold $M$ is said to be non-parabolic if it admits a positive Green's function. Otherwise, $M$ is said to be parabolic. 
For the sake of future reference, we will outline the construction procedure in [69] for $G(x, y)$. Let $p \in M$ be a fixed point and $\left\{\Omega_{i}\right\}$ be a compact exhaustion of $M$ satisfying

$$
\{p\} \subset \Omega_{1} \subset \cdots \subset \Omega_{i} \subset \cdots \subset M
$$

and

$$
\cup_{i} \Omega_{\imath}=M \text {. }
$$

Let $G_{i}(p, \cdot)$ be the positive Dirichlet Green's function on $\Omega_{i}$ with pole at $p$. The fact that

$$
\Omega_{\imath} \subset \Omega_{\jmath}
$$

for $i \leq j$ and the maximum principle implies that

$$
G_{i}(p, \cdot) \leq G_{j}(p, \cdot) .
$$

In particular, if $G_{i}(p, \cdot)$ monotonically converges to some function $G(p, \cdot)$, then $G$ is a positive Green's function, and hence $M$ is non-parabolic. In this case, one checks readily that $G$ is the minimal positive Green's function. The minimality property determines $G$ uniquely.

In the event that $G_{i}(p, \cdot) \nearrow \infty$, by defining

$$
a_{\imath}=\sup _{\partial B_{p}(1)} G_{i}(p, \cdot)
$$

one can show that $G_{i}(p, \cdot)-a_{i}$ converges to some function $G(p, \cdot)$. This function will indeed be a Green's function which changes sign and, in this case, $M$ is parabolic. From this construction, one sees that

$$
G(p, \cdot) \leq 0
$$

on $M \backslash B_{p}(1)$. Note that $G$ is not unique and may depend on the choice of the compact exhaustion.

Let us now examine the situation when $G_{i}(p, \cdot)$ converges to a positive Green's function. It was shown [69] that this occurs if and only if there exists a harmonic function $h$ defined on $M \backslash B_{p}(1)$ with the property that

$$
h=1 \text { on } \partial B_{p}(1)
$$

and

$$
\inf _{M \backslash B_{p}(1)} h=0 .
$$

To understand the existence of $h$, we consider the corresponding problem on annuli of the form $A_{p}(1, r)=B_{p}(r) \backslash B_{p}(1)$. For each $r>1$, let $h_{r}$ be the harmonic function defined on $A_{p}(1, r)$ with the properties that

$$
h_{r}=1 \text { on } \partial B_{p}(1)
$$


and

$$
h_{r}=0 \text { on } \partial B_{p}(r) .
$$

Clearly, $h_{r}$ is the minimizer for the Dirichlet integral

$$
\int_{A_{p}(1, r)}|\nabla f|^{2}
$$

among all functions in the space

$$
H_{r}=\left\{f \in H_{1,2}\left(A_{p}(1, r)\right) \mid f=1 \text { on } \partial B_{p}(1), f=0 \text { on } \partial B_{p}(r)\right\} .
$$

If we define

$$
E(r)=\inf _{H_{r}} \int_{A_{p}(1, r)}|\nabla f|^{2}=\int_{A_{p}(1, r)}\left|\nabla h_{r}\right|^{2}
$$

then clearly $H_{r} \subset H_{R}$ for $r \leq R$. Hence $E(r)$ is a monotonically nonincreasing function of $r$. Due to the boundary conditions, the sequence $h_{r}$ satisfies $h_{r} \leq h_{R}$ for $r \leq R$. The fact that $h_{r} \leq 1$ because of the maximum principle implies that the sequence $\left\{h_{r}\right\}$ converges uniformly on compact subsets to a harmonic function $h_{\infty}$. Moreover, $h_{\infty}$ has the property that

$$
h_{\infty}=1 \text { on } \partial B_{p}(1) .
$$

Clearly, unless $h_{\infty}$ is identically constant 1 , the function

$$
h=\frac{h_{\infty}-\inf h_{\infty}}{1-\inf h_{\infty}}
$$

will be the desired harmonic function we wish to construct.

We now claim that $h_{\infty}$ is the constant function 1 if and only if $E(r) \searrow 0$. Indeed, using the fact that $h_{r}$ is harmonic and the boundary conditions, we can rewrite the integral

$$
\begin{aligned}
E(r) & =\int_{A_{p}(1, r)}\left|\nabla h_{r}\right|^{2} \\
& =\int_{\partial B_{p}(r)} h_{r} \frac{\partial h_{r}}{\partial \nu}-\int_{\partial B_{p}(1)} h_{r} \frac{\partial h_{r}}{\partial \nu} \\
& =-\int_{\partial B_{p}(1)} \frac{\partial h_{r}}{\partial \nu} .
\end{aligned}
$$

Hence the strong maximum principle asserts that $h_{\infty}$ is identically constant if and only if $E(r) \searrow 0$. In particular, this implies that $E(r) \searrow 0$ if and only if $M$ is parabolic. The quantity

$$
\lim _{r \rightarrow \infty} E(r)
$$


is sometimes called the capacity of $M$ at infinity. With this equivalent condition for parabolicity, Royden's theorem [92] follows immediately.

Definition 2.3. A manifold $M$ is said to be quasi-isometric to another manifold $N$ if there exists a diffeomorphism $\phi: M \rightarrow N$ and a constant $C>0$, such that,

$$
C^{-1} d s_{M}^{2} \leq \phi^{*}\left(d s_{N}^{2}\right) \leq C d s_{M}^{2} .
$$

Theorem 2.4 (Royden [92]). Let $M$ be quasi-isometric to $N$. Then $M$ is parabolic if and only if $N$ is parabolic.

Definition 2.5. An end $E$ is said to be a non-parabolic end if it admits a positive Green's function with Neumann boundary condition on $\partial E$. Otherwise, it is said to be a parabolic end. We will denote $\Pi_{0}^{\infty}(M)$ to be the number of parabolic ends of $M$.

From the construction of [69] outlined above, one verifies that $M$ is non-parabolic if and only if it has a non-parabolic end. Indeed, if $E$ is a non-parabolic end, then it admits a Neumann Green's function $G(x, y)$. For a fixed $x \in E$, the strong maximum principle asserts that $G(x, \cdot)$ must be positive on $\partial E$. If we define

$$
g=\min \{G(x, \cdot), a\}
$$

for some sufficiently large constant $a>0$, then $g$ is a positive superharmonic function define on $E$ with

$$
\inf _{E} g=0
$$

and

$$
\inf _{\partial E} g=b>0
$$

for some constant $b$. Clearly, the function $b^{-1} g$ can be used as a barrier to solve for a positive harmonic function on $E$ with

$$
h=1 \text { on } \partial E
$$

and

$$
\inf _{E} h=0 .
$$

The existence of $h$ implies $M$ is non-parabolic as indicated above.

Conversely, if $M$ admits a positive Green's function then the minimal positive Green's function will have the property that

$$
\inf _{M} G(x, \cdot)=0 .
$$


Let $E$ be an end with respect to some compact set containing $x$ such that

$$
\inf _{E} G(x, \cdot)=0 .
$$

Clearly, the above construction together with $\left.G(x, \cdot)\right|_{E}$ can be used to construct a positive Neumann Green's function on $E$.

It is useful to point out that Nakai [90] (also see [91]) showed at if $M$ is parabolic then there exists a Green's function $G(p, \cdot)$ with the property that

$$
G(p, x) \rightarrow-\infty \text { as } x \rightarrow \infty .
$$

\section{Geometric criteria for parabolicity}

Though the definition of parabolicity is purely analytical, in some incidents, there are geometric description of parabolicity. It was first pointed out by Cheng and Yau [26] that if the volume growth of $M$ satisfies

$$
V_{p}\left(r^{2}\right) \leq C r^{2}
$$

for some constant $C>0$, then $M$ must be parabolic. The sharp condition was proved by Ahlfors for dimension 2, and later independently by Grigor'yan [43], [44] and Varopoulos [103] for all dimensions, that a necessary condition for a manifold to be non-parabolic is that there exists $p \in M$, such that, the volume $V_{p}(t)$ of geodesic ball centered at $p$ of radius $t$ satisfies the growth condition

$$
\int_{1}^{\infty} \frac{t d t}{V_{p}(t)}<\infty .
$$

Observe that this property holds at one point if and only if it holds at all points of $M$. Moreover, this condition is clearly invariant under quasiisometry. The obvious question is to determine if this condition is also sufficient. Unfortunately, the following example of Greene (see [103]) indicated that this is not true in general.

Example. Let $M$ be $\mathbb{R}^{2}$ endowed with the metric of the form

$$
d s^{2}=\left\{\begin{array}{rll}
y^{-2}\left(d x^{2}+d y^{2}\right) & \text { for } & y \geq 2 \\
f(y)\left(d x^{2}+d y^{2}\right) & \text { for } & 0 \leq y \leq 2 \\
d x^{2}+d y^{2} & \text { for } & y \leq 0,
\end{array}\right.
$$

where $f$ is any smooth function satisfying $f(0)=1$ and $f(2)=1 / 4$. This manifold is obviously parabolic because it is conformally equivalent to 
the standard flat metric on $\mathbb{R}^{2}$. However, direct computation shows that (3.1) holds.

An interesting phenomenon is that for manifolds with non-negative Ricci curvature, condition (3.1) is also sufficient for non-parabolicity.

Theorem 3.1 (Varopoulos [102]). If $M$ has non-negative Ricci curvature, then $M$ is non-parabolic if and only if

$$
\int_{1}^{\infty} \frac{t d t}{V_{p}(t)}<\infty
$$

for some $p \in M$, where $V_{p}(t)$ is the volume of geodesic ball centered at $p$ of radius $t$.

In fact, in the case of non-negative Ricci curvature, one can estimate the Green's function by the volume growth.

Theorem 3.2 (Li-Yau [79]). If $M$ has non-negative Ricci curvature, then there exists positive constants $C_{1}$ and $C_{2}$, such that, the minimal positive Green's function satisfies

$$
C_{1} \int_{\rho(x, y)}^{\infty} \frac{t d t}{V_{x}(t)} \leq G(x, y) \leq C_{2} \int_{\rho(x, y)}^{\infty} \frac{t d t}{V_{x}(t)},
$$

where $\rho(x, y)$ denotes the geodesic distance between $x$ and $y$.

In 1995, Li-Tam managed to prove that the volume growth condition is sufficient for non-parabolicity for a larger class of manifolds.

Theorem 3.3 (Li-Tam [73]). Let us assume that there is a constant $C_{1}>0$ such that the Ricci curvature of $M$ satisfies

$$
\operatorname{Ric}_{M}(x) \geq-C_{1} \rho^{-2}(x)
$$

for all $x \in M$. Assume that there exists $p \in M$ and $C_{2}>0$, such that, the volume comparison condition

$$
V_{p}(R) \leq C_{2} V_{x}(R / 2),
$$

is satisfied for all $x \in \partial B_{p}(R)$, then $M$ is non-parabolic if and only if

$$
\int_{1}^{\infty} \frac{t d t}{V_{p}(t)}<\infty
$$


Corollary 3.4. If $M$ has non-negative Ricci curvature near infinity and finite first Betti number, then $M$ is non-parabolic if and only if

$$
\int_{1}^{\infty} \frac{t d t}{V_{p}(t)}<\infty
$$

for some $p \in M$.

Corollary 3.5. If $M$ is quasi-isometric to a manifold satisfying the assumption of Theorem 3.3, then $M$ is non-parabolic if and only if

$$
\int_{1}^{\infty} \frac{t d t}{V_{p}(t)}<\infty
$$

for some $p \in M$.

In [73], the authors obtained estimates for the Green's function on manifolds satisfying the hypothesis of Theorem 3.3. However, the estimates are not as clean as those of Theorem 3.4. Recently, ColdingMinicozzi [31] showed that if $M^{n}$ with $n \geq 3$ has non-negative Ricci curvature and maximal volume growth then the Green's function has an asymptotic limit. In a joint work [74] of Tam, Wang, and the author, they gave a short proof of the asymptotic limit and also gave sharp upper and lower bounds for $G$. In this case, maximal volume growth means that there exists $p \in M$ such that

$$
\liminf _{r \rightarrow \infty} r^{-n} V_{p}(r)>0
$$

Bishop comparison theorem implies that, in fact,

$$
\theta_{p}(r)=r^{-n} V_{p}(r)>0
$$

is a monotonically non-increasing function of $r$. Also, it is easy to see that if

$$
\theta=\lim _{r \rightarrow \infty} \theta_{p}(r)
$$

then $\theta$ is independent of $p$.

Theorem 3.6 (Li-Tam-Wang [74]). Let $M$ be a complete manifold with non-negative Ricci curvature of dimension at least 3. Assume that $M$ has maximal volume growth, and let $\rho$ be the distance function to the point $p \in M$. For any $\delta>0$, there exists a constant $C>0$ depending only on $n$ and $\theta$, so that the minimal positive Green's function on $M$ satisfies

$$
\begin{aligned}
(1+9 \delta)^{1-\frac{n}{2}} & \frac{\rho^{2-n}(x)}{n(n-2) \theta_{p}(\delta \rho(x))} \\
& \leq G(p, x) \\
& \leq(1+C(\delta+\beta))(1-\delta)^{1-\frac{n}{2}} \frac{\rho^{2-n}(x)}{n(n-2) \theta}
\end{aligned}
$$


where

$$
\beta=\delta^{-2 n} \max _{r \geq(1-\delta) \rho(x)}\left\{1-\frac{\theta_{p}(r)}{\theta_{p}\left(\delta^{2 n+1} r\right)}\right\} .
$$

In particular,

$$
\lim _{x \rightarrow \infty} \rho^{n-2}(x) G(p, x)=\frac{1}{n(n-2) \theta}
$$

Let us consider the special case when $M$ is a complete manifold with a rotationally symmetric metric with respect to a point $p \in M$. If $A_{p}(t)$ denotes the area of $\partial B_{p}(t)$, then let us assume that

$$
\int_{1}^{\infty} \frac{d t}{A_{p}(t)}<\infty
$$

In this case, $M$ is non-parabolic and the minimal positive Green's function with the pole at $p$ is given by

$$
G(p, x)=\int_{\rho(p, x)}^{\infty} \frac{d t}{A_{p}(t)} .
$$

Indeed, using the fact that $A_{p}(t)$ is asymptotically

$$
A_{p}(t) \sim n \omega_{n} t^{n-1}
$$

as $t \rightarrow 0$, where $\omega_{n}$ denotes the volume of the Euclidean unit $n$-ball, we verify that

$$
\int_{\rho(p, x)}^{\infty} \frac{d t}{A_{p}(t)} \sim \frac{1}{n(n-2) \omega_{n}} \rho^{2-n}(p, x)
$$

as $x \rightarrow p$. Also, since the metric is rotationally symmetric, the Laplacian in terms of polar coordinates can be written as

$$
\Delta=\frac{\partial^{2}}{\partial r^{2}}+\frac{A_{p}^{\prime}}{A_{p}} \frac{\partial}{\partial r}
$$

hence

$$
\Delta\left(\int_{\rho(p, x)}^{\infty} \frac{d t}{A_{p}(t)}\right)=0
$$

for $x \neq p$, and (3.3) is verified. In fact, a similar computation will confirm that the function

$$
\int_{1}^{\rho(p, x)} \frac{d t}{A_{p}(t)}
$$


is a Green's function on a rotationally symmetric manifold regardless of parabolicity. In case (3.2) holds, then (3.4) differs from (3.3) by a additive constant. If (3.2) is not valid, then (3.4) is still a Green's function and $M$ is parabolic. Notice that if $M$ satisfies some non-negativity assumption on the Ricci curvature, then one can show that $t A_{p}(t)$ is equivalent to $V_{p}(t)$, which explains the validity of Theorem 3.3.

In a recent works of Holopainen [50] and Holopainen-Koskela [51], the authors gave a criteria upon which the condition (3.1) is equivalent to non-parabolicity. In particular, one criterion has the property that it is localized on a cone neighborhood of a geodesic ray.

Theorem 3.7 (Holopainen-Koskela [51]). Let $M$ be a complete manifold. Suppose there exists a geodesic ray $\gamma:[0, \infty) \rightarrow M$ satisfying the following two properties:

- There exists a constant $C_{1}>0$, such that, for all $t>0$ and for all geodesic ball $B_{x}(2 r) \subset B_{\gamma(t)}\left(\frac{t}{2}\right)$ the volume doubling condition

$$
C_{1} V_{x}(r) \geq V_{x}(2 r)
$$

is satisfied.

- There exists a constant $C_{2}>0$, such that, for all $t>0$ and for all $B_{x}(2 r) \subset B_{\gamma(t)}\left(\frac{t}{2}\right)$ the Poincare inequality

$$
C_{2} r\left(\int_{B_{x}(2 r)}|\nabla f|^{2}\right)^{f r a c 12} \geq \inf _{k \in \mathbb{R}} \int_{B_{x}(r)}|f-\bar{f}| .
$$

is satisfied for all $f \in H_{1,2}\left(B_{x}(r)\right)$ with $\bar{f}=V_{x}(r)^{-1} \int_{B_{x}(r)} f$.

The manifold $M$ is non-parabolic if and only if

$$
\int_{1}^{\infty} \frac{t d t}{V_{p}(t)}<\infty
$$

for some $p \in M$.

We would like to remark that the authors actually proved a more general version of this theorem which holds for the $p$-Laplacian.

\section{A basic theorem on harmonic functions}

In this section, we will indicate that various spaces of harmonic functions will play certain roles in reflecting the topology of the underlying manifold. 
Definition 4.1. Define $\mathcal{H}_{D}^{\infty}(M)$ to be the space of bounded harmonic functions with finite Dirichlet integral on $M$.

Definition 4.2. Define $\mathcal{H}^{\infty}(M)$ to be the space of bounded harmonic functions on $M$.

Definition 4.3. Define $\mathcal{H}^{+}(M)$ to be the space spanned by the set of positive harmonic functions on $M$.

Definition 4.4. Define $\mathcal{H}^{\prime}(M)$ to be the space spanned by the set of harmonic functions which are bounded on one side at each end of $M$. More precisely, a harmonic function, $f$, is bounded on one side at each end if there exists a compact set $D \subset M$ such that $f$ is either bounded from above or from below when restricted to each end with respect to $D$.

It follows directly from the definitions that these spaces satisfy the monotonic relations

$$
\{\text { constants }\} \subset \mathcal{H}_{D}^{\infty}(M) \subset \mathcal{H}^{\infty}(M) \subset \mathcal{H}^{+}(M) \subset \mathcal{H}^{\prime}(M) .
$$

In particular, their respective dimensions satisfy

$$
1 \leq \operatorname{dim} \mathcal{H}_{D}^{\infty}(M) \leq \operatorname{dim} \mathcal{H}^{\infty}(M) \leq \operatorname{dim} \mathcal{H}^{+}(M) \leq \operatorname{dim} \mathcal{H}^{\prime}(M) .
$$

Observe that if $M$ has only one end, then $\mathcal{H}^{+}(M)=\mathcal{H}^{\prime}(M)$.

Definition 4.5. A manifold is said to have the strong Liouville property if it does not admit any non-constant positive harmonic function, i.e., $\operatorname{dim} \mathcal{H}^{+}(M)=1$.

Definition 4.6. A manifold is said to have the Liouville property if it does not admit any non-constant bounded harmonic function, i.e., $\operatorname{dim} \mathcal{H}^{\infty}(M)=1$.

An interesting, but unrelated fact concerning the space $\mathcal{H}_{D}^{\infty}(M)$ is a theorem of Sario-Schiffer-Glasner [95]. It asserts that if $M$ admits a non-constant harmonic function with finite Dirichlet integral, then it must also admits a non-constant bounded harmonic function with finite Dirichlet integral. We are now ready to state the theorem which relates the dimension of these spaces of harmonic functions to $\pi_{0}^{\infty}(M)$ and $\Pi_{0}^{\infty}(M)$.

Theorem 4.7 (Li-Tam [72]). Let $M$ be any complete Riemannian manifold without boundary. The the number of ends, $\pi_{0}^{\infty}(M)$, of $M$ satisfies the upper bound

$$
\pi_{0}^{\infty}(M) \leq \operatorname{dim} \mathcal{H}^{\prime}(M)
$$


If $M$ is non-parabolic, then we have the improved estimate

$$
\pi_{0}^{\infty}(M) \leq \operatorname{dim} \mathcal{H}^{+}(M)
$$

and the number of non-parabolic ends, $\Pi_{0}^{\infty}(M)$, satisfies the bound

$$
\Pi_{0}^{\infty}(M) \leq \operatorname{dim} \mathcal{H}_{D}^{\infty}(M) .
$$

We should point out that the last case of Theorem 4.7 also follows from the work of Grigor'yan [46], where he related $\operatorname{dim} \mathcal{H}_{D}^{\infty}(M)$ to the number disjoint of D-massive sets. At this point, perhaps it is useful to consider a few examples so we have a better understanding of this theorem.

Example 1. Let $M=\sharp k\left(\mathbb{R}^{n}\right)$ be the connected sum of $k$ copies of $\mathbb{R}^{n}$. If $n=2$, then $M$ is parabolic. In this case,

$$
\pi_{0}^{\infty}(M)=k=\operatorname{dim} \mathcal{H}^{\prime}(M) .
$$

If $n \geq 3$, then $M$ is non-parabolic, and

$$
\pi_{0}^{\infty}(M)=\Pi_{0}^{\infty}(M)=k .
$$

Moreover,

$$
\operatorname{dim} \mathcal{H}^{\prime}(M)=\operatorname{dim} \mathcal{H}^{+}(M)=\operatorname{dim} \mathcal{H}_{D}^{\infty}(M)=k .
$$

In any event, Theorem 4.7 is sharp.

Example 2. Let $M$ be the hyperbolic plane $\mathbb{H}^{2}$. In this case,

$$
\operatorname{dim} \mathcal{H}^{\prime}(M)=\operatorname{dim} \mathcal{H}^{+}(M)=\operatorname{dim} \mathcal{H}_{D}^{\infty}(M)=\infty .
$$

However, $M$ is non-parabolic and has only 1 end.

Example 3. Recall that a well-known theorem of Yau asserts that:

Theorem 4.8 (Yau [107]). If $M$ has non-negative Ricci curvature, then $M$ has the strong Liouville property.

On the other hand, the splitting theorem of Cheeger-Gromoll implies that:

Theorem 4.9 (Cheeger-Gromoll [21]). If $M$ has non-negative Ricci curvature, then either $M=N \times \mathbb{R}$, for some compact manifold $N$ with non-negative Ricci curvature, or $M$ has only 1 end. 
Using these two theorems, we will analyze the situation when $M$ has non-negative Ricci curvature. First, let us consider the case when $M=N \times \mathbb{R}$. In this case, clearly $M$ is parabolic according to the volume growth condition (3.1) for non-parabolicity. One also checks easily that the space $\mathcal{H}^{\prime}(M)$ is spanned by the constant function 1 and the function $t \in \mathbb{R}$. Hence

$$
\operatorname{dim} \mathcal{H}^{\prime}(M)=2=\pi_{0}^{\infty}(M) .
$$

The remaining case is when $M$ has only 1 end. This implies that

$$
\mathcal{H}^{\prime}(M)=\mathcal{H}^{+}(M)
$$

from the definition of $\mathcal{H}^{\prime}(M)$. In this case, Yau's result implies that

$$
\operatorname{dim} \mathcal{H}^{\prime}(M)=\operatorname{dim} \mathcal{H}^{+}(M)=1 .
$$

Hence whether $M$ is parabolic or not, Theorem 4.7 is sharp for manifolds with non-negative Ricci curvature.

To give a more quantitative description of Theorem 4.7, let us first consider the case when $M$ is parabolic. For any compact subset $D \subset M$, let $\left\{e_{i}\right\}_{i=1}^{s}$ be the set of all ends. For each $i \neq 1$, there exists a harmonic function $f_{i}$ satisfying

$$
\begin{aligned}
& f_{i}(x) \rightarrow-\infty \quad \text { as } \quad x \rightarrow e_{1}(\infty), \\
& f_{i}(x) \rightarrow \infty \quad \text { as } \quad x \rightarrow e_{i}(\infty),
\end{aligned}
$$

and $f_{i}$ is bounded on $e_{j}$ for all $j \neq 1, i$. The notation $x \rightarrow e_{i}(\infty)$ means that $x \rightarrow \infty$ and $x \in e_{i}$. One checks readily that the set $\left\{f_{i}\right\}_{i=2}^{s}$ together with the constant function form a linearly independent set. Hence, $s \leq$ $\operatorname{dim} \mathcal{H}^{\prime}(M)$ and the first assertion of Theorem 4.7 follows because $D$ is arbitrary.

When $M$ is non-parabolic, for any compact subset $D$, let $\left\{e_{i}\right\}_{i=1}^{s}$ be the set of parabolic ends with respect to $D$ and $\left\{E_{\alpha}\right\}_{\alpha=1}^{\ell}$ be the set of non-parabolic ends with respect to $D$. If $s>0$, then for each $1 \leq i \leq s$, there exists a positive harmonic function $g_{i}$ satisfying

$$
\begin{gathered}
\inf _{E_{\alpha}} g_{i}(x)=0 \quad \text { for all } \quad \alpha, \\
g_{i}(x) \rightarrow \infty \quad \text { as } \quad x \rightarrow e_{i}(\infty),
\end{gathered}
$$

and $g_{i}$ is bounded on $e_{k}$ for all $k \neq i$. Also, for any $1 \leq \alpha \leq \ell$, there exists a bounded harmonic function $h_{\alpha}$ with the properties that

$$
\sup _{E_{\alpha}} h_{\alpha}=1,
$$




$$
\inf _{E_{\beta}} h_{\alpha}=0 \quad \text { for } \quad \beta \neq \alpha,
$$

and $h_{\alpha}$ is bounded on $e_{i}$ for all $1 \leq i \leq s$. Similarly to the parabolic case, the existence of these harmonic functions implies the inequality asserted in Theorem 4.7.

\section{Historical background}

Before we proceed to discuss various applications of Theorem 4.7, perhaps it is useful to point out some historical aspects that lead to the development of the theorem. In his fundamental paper [107], Yau introduced the method of gradient estimate to prove Theorem 4.8. Right after this work, Cheng-Yau [26] provided a local argument for the gradient estimate which will become a basic technique and a starting point for the theory of harmonic functions in years to come. The version of the gradient estimate that is related to the content of this article is as follows:

Theorem 5.1 (Cheng-Yau [26]). Let $M$ be a manifold with boundary, $\partial M$. Suppose $p \in M$ and $r>0$ such that the geodesic ball $B_{p}(r)$ centered at $p$ of radius $r$ satisfies $B_{p}(r) \cap \partial M=\emptyset$. If $f$ is a positive harmonic function defined on $B_{p}(r)$, then for any $0<\alpha<1$, there exists a constant $C>0$ depending only on $n=\operatorname{dim} M$ and $\alpha$, such that,

$$
|\nabla f|(x) \leq C r^{-1} f(x)
$$

for all $x \in B_{p}(\alpha r)$. In particular,

$$
f(x) \leq C f(y)
$$

for all $x, y \in B_{p}(\alpha r)$.

In 1987, in an attempt to understand and generalize Yau's strong Liouville theorem to a larger class of manifold, the author and Luenfai Tam considered manifolds which behave like a manifold with nonnegative Ricci curvature. The most elementary situation which we considered is the case when $M$ is a connected sum of $k$ copies of $\mathbb{R}^{n}$ as given by Example 1 of the previous section. The example indicated that the validity of Yau's theorem hinges on Theorem 4.9, even though Yau's proof is completely independent of Theorem 4.9. Also, the fact that the manifold is Euclidean at each end allows us to use the explicit form of the Green's function as barriers. In fact, modeling on Example 1, Li-Tam [68] successfully determined the spaces of bounded and positive 
harmonic functions on a manifold with non-negative sectional curvature near infinity.

Theorem 5.2 (Li-Tam [68]). Let $M$ be a complete manifold with non-negative sectional curvature near infinity. It follows that $M$ must have finitely many ends. Hence there exists $p \in M$ and $r>0$ such that the number of ends with respect to $B_{p}(r)$ is precisely $\pi_{0}^{\infty}(M)$. An end $E$ is non-parabolic if and only if

$$
\int_{r}^{\infty} \frac{t d t}{V_{E}(t)}<\infty
$$

Suppose $\left\{e_{i}\right\}_{l=1}^{s}$ as the set of ends satisfying the volume growth condition

$$
\int_{r}^{\infty} \frac{t d t}{V_{e_{i}}(t)}=\infty
$$

where $V_{e_{i}}(t)$ denotes the volume of the set $B_{p}(t) \cap e_{i}$. Also, let $\left\{E_{\alpha}\right\}_{\alpha=1}^{\ell}$ be the set of ends satisfying the volume growth condition

$$
\int_{r}^{\infty} \frac{t d t}{V_{E_{\alpha}}(t)}<\infty
$$

In particular, we have

$$
\Pi_{0}^{\infty}(M)=\ell \quad \text { and } \quad \pi_{0}^{\infty}(M)=s+\ell .
$$

If $\Pi_{0}^{\infty}(M)=0$ then $\operatorname{dim} \mathcal{H}^{+}(M)=1$. If $\Pi_{0}^{\infty}(M)>0$, then for each $e_{i}$ there exists a positive harmonic function $g_{i}$ satisfying

$$
\begin{aligned}
g_{i}(x) \rightarrow 0 \quad \text { as } & x \rightarrow E_{\alpha}(\infty) \quad \text { for all } \quad \alpha, \\
& g_{i}(x) \rightarrow \infty \quad \text { as } \quad x \rightarrow e_{i}(\infty),
\end{aligned}
$$

and $g_{i}$ is bounded on $e_{k}$ for all $k \neq i$. Also, for any $1 \leq \alpha \leq \ell$, there exists a bounded harmonic function $h_{\alpha}$ with the properties that

$$
\begin{array}{llll}
h_{\alpha}(x) \rightarrow 1 & \text { as } & & x \rightarrow E_{\alpha}(\infty), \\
h_{\alpha}(x) \rightarrow 0 & \text { as } & x \rightarrow E_{\beta}(\infty)
\end{array}
$$

for all $\beta \neq \alpha$, and $h_{\alpha}$ is bounded on $e_{i}$ for all $1 \leq i \leq s$. Moreover, the set $\left\{h_{\alpha}\right\}_{\alpha=1}^{\ell}$ spans the space of bounded harmonic functions denoted by $\mathcal{H}^{\infty}(M)$, and the set $\left\{h_{\alpha}\right\}_{\alpha=1}^{\ell} \cup\left\{g_{i}\right\}_{i=1}^{s}$ spans the space $H^{+}(M)$. In particular,

$$
\Pi_{0}^{\infty}(M)=\mathcal{H}^{\infty}(M) \quad \text { and } \quad \pi_{0}^{\infty}(M)=\mathcal{H}^{+}(M) .
$$


The reason that sectional curvature was assumed is because some of the arguments used in proving Theorem 1.1 can be used to restrict the topology and geometry at infinity of these manifolds. In particular, the fact that these manifolds have finitely many ends, with each end homeomorphic to a product space $N \times[0, \infty)$, is extensively used in the proof. In the paper [68], the authors raised the question that if we replace the sectional curvature assumption in Theorem 5.2 by the Ricci curvature, to what extend will the consequences of the theorem still remain valid. The first obstacle in proving this is to determine if manifolds with non-negative Ricci curvature near infinity has only finitely many ends.

Around the same time, Donnelly [37] proved that the space of bounded harmonic functions, $\mathcal{H}^{\infty}(M)$, on a manifold with non-negative Ricci curvature near infinity must be finite. Later, in an unpublished work, Cheng showed that if $M$ has non-negative Ricci curvature outside a set $D$ with diameter $a$, and if the Ricci curvature is bounded from below by $-K$ on $D$ for some $K>0$, then there exists a constant $C(n, a \sqrt{K})>0$ depending only on $n$ and $a \sqrt{K}$ such that

$$
\operatorname{dim} \mathcal{H}^{\infty}(M) \leq C(n, a \sqrt{K}) .
$$

He also proved that $\mathcal{H}^{+}(M)$ must be finite dimensional. In view of these developments, if Theorem 5.2 holds for manifolds with non-negative Ricci curvature near infinity, then it will imply that $M$ has finitely many ends if $M$ is non-parabolic. In fact, this provides the motivation behind Theorem 4.7.

\section{Applications to Riemannian geometry}

Theorem 4.7 allows us to estimate the number of ends, by estimating $\operatorname{dim} \mathcal{H}^{\prime}(M)$.

Theorem 6.1 (Li-Tam [72]). Assume that $M$ has asymptotically non-negative Ricci curvature as defined in $\S 1$. Then there exists a constant $C(\alpha, n)>0$ depending only on $\alpha$ and $n=\operatorname{dim} M$ such that

$$
\pi_{0}^{\infty}(M) \leq C(\alpha, n)
$$

For the special case when $M$ has non-negative Ricci curvature outside some compact set $D$, then the estimate on $\pi_{0}^{\infty}(M)$ takes the form

$$
\pi_{0}^{\infty}(M) \leq C_{1} \exp \left(C_{2} a \sqrt{K}\right)+1
$$


where $a$ is the diameter of $D,-K<0$ is the lower bound of the Ricci curvature on $D$, and $C_{1}$ and $C_{2}$ are constants depending only on $n$.

We would like to point out that independently Cai [17] used a Riemannian geometric method to prove a slightly weaker estimate for the case when $M$ has non-negative Ricci curvature near infinity. Later, CaiColding-Yang [18] refined Cai's argument and showed that if $a \sqrt{K}$ is sufficiently small, then $M$ has at most 2 ends. This can be viewed as a generalization of the consequence of the splitting theorem (Theorem 4.9). Using some of the argument of Cai, Liu [82] also proved a ball covering property for these manifolds.

Theorem 6.2 (Liu [82]). Let $M$ be a complete manifold with nonnegative Ricci curvature outside a compact set $D \subset B_{p}(a)$. Let $-K<0$ be the lower bound of the Ricci curvature on D. For any $\mu>0$, there exists a constant $C(n, a \sqrt{K}, \mu)>0$, such that, for any $r>0$ there exists $a$ set of points $\left\{p_{1}, \ldots, p_{k}\right\} \subset \overline{B_{p}(r)}$ with $k \leq C(n, a \sqrt{K}, \mu)$ satisfying

$$
\overline{B_{p}(r)} \subset \cup_{i=1}^{k} B_{p_{i}}(\mu r) .
$$

Observe that the ball covering theorem implies that

$$
\pi_{0}^{\infty}(M) \leq C(n, a \sqrt{K}, 1 / 2) .
$$

It is interesting to point out that it is still not known if the ball covering property holds for manifolds with asymptotically non-negative Ricci curvature. Note that for a non-parabolic manifold, in order to prove that the inequality

$$
\operatorname{dim} \mathcal{H}_{D}^{\infty}(M) \geq \Pi_{0}^{\infty}(M)
$$

is indeed an equality, it is necessary to show that any bounded harmonic function must have a unique infinity behavior up to a scalar multiple at each non-parabolic end. For example, for the case when $M$ has nonnegative sectional curvature near infinity, the authors [68] showed that a bounded harmonic function must be asymptotically constant at infinity of each non-parabolic end. One way to show this is to develop a spherical Harnack inequality, which asserts that there is a constant depending only on $M$ such that, if $f$ is a positive harmonic function defined on $E$ then

$$
f(x) \leq C f(y)
$$

for all $x, y \in \partial B_{p}(r) \cap E$. This type of inequality allows us to conclude that if

$$
\liminf _{x \rightarrow E(\infty)} f=0,
$$


then

$$
\lim _{x \rightarrow E(\infty)} f=0
$$

If $M$ has non-negative Ricci curvature on $E$ then using Theorem 5.1, we conclude that

$$
f(x) \leq C f(y)
$$

for $x \in \partial B_{p}(r)$ and $y \in B_{p}(r / 2)$. Hence, if we know that $\partial B_{p}(r) \cap E$ is connected, the ball covering property implies that one can iterate the inequality at most $C(n, a \sqrt{K}, 1 / 2)$ times and obtain the spherical Harnack inequality. It turns out that if we assume $M$ has finite first Betti number then one can show the basic connectedness of $\partial B_{p}(r) \cap E$. This line of argument yield the following theorem:

Theorem 6.3 (Li-Tam [73]). Let $M$ be a complete manifold with non-negative Ricci curvature near infinity. Suppose the first Betti number of $M$ is finite, then all the inequalities of Theorem 4.7 become equalities. In particular,

$$
\pi_{0}^{\infty}(M)=\operatorname{dim} \mathcal{H}^{\prime}(M),
$$

and if $M$ is non-parabolic then

$$
\pi_{0}^{\infty}(M)=\operatorname{dim} \mathcal{H}^{+}(M)=\operatorname{dim} \mathcal{H}^{\prime}(M)
$$

and

$$
\Pi_{0}^{\infty}(M)=\operatorname{dim} \mathcal{H}_{D}^{\infty}(M)
$$

We do not know of a complete manifold with non-negative Ricci curvature near infinity, but have infinite first Betti number. It is plausible that the finiteness of $b_{1}(M)$ is a consequence of the curvature assumption.

We should also point out that, in proving Theorem 3.3, the authors [73] proved that the ball covering property holds on a manifold satisfying the hypothesis of Theorem 3.3. However, it is not known that the volume comparison condition asserted in Theorem 3.3 holds even on manifolds with non-negative Ricci curvature near infinity and has only 1 end.

Theorem 4.7 can also be applied to study stable minimal hypersurfaces. In 1976, in their study of stable minimal hypersurfaces, Schoen and Yau [97] showed that a complete, oriented, stable minimal hypersurface $M^{n}$ in a manifold of non-negative Ricci curvature must have

$$
\operatorname{dim} H_{D}^{\infty}(M)=1
$$


Exploiting this fact, Cao, Shen, and Zhu proved that such a manifold must have only 1 end.

Theorem 6.4 (Cao-Shen-Zhu [19]) If $M^{n}(n \geq 3)$ be a complete, oriented, stable minimal hypersurface in $\mathbb{R}^{n+1}$, then

$$
\pi_{0}^{\infty}(M)=1
$$

Their argument used the Sobolev inequality of Michael-Simon [85] to conclude that each end of $M$ must be non-parabolic. Hence one can apply the estimate to conclude that

$$
\pi_{0}^{\infty}(M)=\Pi_{0}^{\infty}(M) .
$$

An upshot of their argument is the following general fact on complete manifolds. If a complete manifold $M$ satisfies a Sobolev inequality of the form

$$
\left(\int_{B_{p}(r)}|f|^{2 p}\right)^{\frac{1}{p}} \leq C \int_{B_{p}(r)}|\nabla f|^{2}
$$

for some constants $C>0, p \geq 1$ and for all $f \in H_{1,2}^{c}\left(B_{p}(r)\right.$, then each end $E$ of $M$ must either have finite volume or be non-parabolic. In particular, using the necessary criteria (3.1) for non-parabolicity, one concludes that either the volume of $E$ is finite or

$$
\int_{1}^{\infty} \frac{t d t}{V_{E}(t)}<\infty
$$

This constitutes a gap phenomenon for the volume growth on manifolds satisfying (6.2). Note that a finite volume end is possible. This can be seen by taking a complete metric on a 2-dimensional annulus which has constant -1 curvature. One can arrange the metric to have finite volume on one end, but infinite volume on the other end. In this case, one verifies easily that (6.2) holds for $p=1$. Also, the manifold is non-parabolic, due to the existence of one non-parabolic end.

\section{Function theory under quasi-isometries}

Recall that Theorem 2.4 asserts that parabolicity is a quasi-isometric invariant. On the other hand, any topological data is certainly invariant under quasi-isometries. Therefore, it is interesting to ask if the dimensions of the spaces $\mathcal{H}^{\prime}, \mathcal{H}^{+}$, and $\mathcal{H}_{D}^{\infty}$ are quasi-isometric invariants. 
An example of Lyons [83] shows that there are manifolds $M$ and $N$ which are quasi-isometric but

$$
\operatorname{dim} \mathcal{H}^{+}(M)=1 \quad \text { and } \quad \operatorname{dim} \mathcal{H}^{\infty}(N)>1 .
$$

On the other hand, Grigor'yan [45], [46] proved that the dimension of the space $\mathcal{H}_{D}^{\infty}$ is invariant under quasi-isometry. This leads us to the question that perhaps there are spaces of harmonic functions $\mathcal{H}_{1}$ and $\mathcal{H}_{2}$ which play the same roles as $\mathcal{H}^{+}$and $\mathcal{H}^{\prime}$ in Theorem 4.7 , but their dimensions are quasi-isometric invariants.

Recall that the De Giorgi-Nash-Moser theory implies that if a manifold $M$ is quasi-isometric to $\mathbb{R}^{n}$, then it must have the strong Liouville property, namely,

$$
\operatorname{dim} \mathcal{H}^{+}(M)=1 .
$$

In view of this Yau conjectured that if a manifold $M$ is quasi-isometric to a manifold with non-negative Ricci curvature then

$$
\operatorname{dim} \mathcal{H}^{+}(M)=1 .
$$

In fact, this was verified by Grigor'yan and Saloff-Coste independently.

Theorem 7.1 (Grigor'yan [47] and Saloff-Coste [93]). Let $M$ be $a$ complete manifold satisfying the following two properties:

- Volume doubling property which asserts that there exists a constant $\eta>0$ depending only on $M$ such that

$$
2^{\eta} V_{p}(r) \geq V_{p}(2 r)
$$

for all $p \in M$ and $r>0$; and

- Weak Poincaré inequality which asserts that there exists a constant $C>0$ depending only on $M$ such that

$$
\int_{B_{p}(2 r)}|\nabla f|^{2} \geq C r^{-2} \inf _{k} \int_{B_{p}(r)}(f-k)^{2}
$$

for all functions $f \in H_{1,2}\left(B_{p}(2 r)\right)$.

Then

$$
\operatorname{dim} \mathcal{H}^{+}(M)=1 .
$$


Since both the volume doubling property and the weak Poincaré inequality are invariants under quasi-isometries, and they both hold for manifolds with non-negative Ricci curvature, this implies Yau's conjecture. Along the same direction, Sung pushed this one step further.

Theorem 7.2 (Sung [99]). Let $M$ be quasi-isometric to a manifold $N$ with non-negative Ricci curvature near infinity. If $M$ has finite first Betti number, then all the inequalities in Theorem 4.7 become equalities. In particular

$$
\operatorname{dim} \mathcal{H}^{\prime}(M)=\operatorname{dim} \mathcal{H}^{\prime}(N)=\pi_{0}^{\infty}(N)=\pi_{0}^{\infty}(N) .
$$

Moreover if $M$ is non-parabolic then

$$
\operatorname{dim} \mathcal{H}^{+}(M)=\operatorname{dim} \mathcal{H}^{+}(N)=\pi_{0}^{\infty}(N)=\pi_{0}^{\infty}(M),
$$

and

$$
\operatorname{dim} \mathcal{H}_{D}^{\infty}(M)=\operatorname{dim} \mathcal{H}_{D}^{\infty}(N)=\Pi_{0}^{\infty}(N)=\Pi_{0}^{\infty}(M) .
$$

A weaker version of isometry was defined by Kanai [55].

Definition 7.3. A map $f: X \rightarrow Y$ between two metric spaces $X$ and $Y$ is a rough isometry if there exists constants $k \geq 1, b>0$, and $c>0$, such that, for all $y \in Y$ there exists $x \in X$ with the properties that

$$
d_{Y}(y, f(x)) \leq c,
$$

and for any $x_{1}, x_{2} \in X$

$$
k^{-1} d_{X}\left(x_{1}, x_{2}\right)-b \leq d_{Y}\left(f\left(x_{1}\right), f\left(x_{2}\right)\right) \leq k d_{X}\left(x_{1}, x_{2}\right)+b .
$$

He studied the effect of function theory under rough isometries for a special class of manifolds.

Definition 7.4. A complete manifold is said to have bounded Ricci geometry if its Ricci curvature is bounded from below and its injectivity radius is strictly positive.

In [55], Kanai showed that if $M$ has bounded Ricci geometry and it is roughly isometric to $\mathbb{R}^{m}$ then $M$ satisfies the strong Liouville property. He [56] also showed that if $M$ is roughly isometric to $N$ and both manifolds have bounded Ricci geometry, then $M$ is parabolic if and only if $N$ is parabolic. In 1993, Holopainen [49] generalized these theorems for the $p$-Laplacian on manifolds with bounded Ricci geometry and finitely 
many ends, all of which are roughly isometric to Euclidean space. Recently Coulhon and Saloff-Coste generalized Kanai's theorem.

Theorem 7.4 (Coulhon-Saloff-Coste [36]). Suppose $f: M \rightarrow N$ is a rough isometry. Assume that there exists a constant $C>0$ such that $f$ satisfies

$$
C^{-1} V_{x}(1) \leq V_{f(x)}(1) \leq C V_{x}(1)
$$

for all $x \in M$. Also, assume that both manifolds have Ricci curvatures bounded from below, then $M$ is parabolic if and only if $N$ is parabolic. Moreover, if $M$ has non-negative Ricci curvature then $N$ satisfies the strong Liouville property.

In fact, the Ricci curvature lower bound can be replaced by the assumption that both manifolds satisfy a local parabolic Harnack inequality. Also the non-negative Ricci curvature assumption can be replaced by a global parabolic Harnack inequality.

In [36], the authors define a rough isometry satisfying (7.3) as an isometry at infinity. An upshot of their analysis is that if a manifold $M$ has Ricci curvature bounded from below, and it is isometric at infinity to a manifold with non-negative Ricci curvature, then $M$ must satisfy (7.1) and (7.2). This fact will revisit in the discussion in $\S 11$.

\section{Applications to Kähler geometry}

In this section, we will discuss various applications of harmonic function theory to Kähler geometry.

Theorem 8.1 (Napier-Ramachandran [91]). Let $M$ be a complete Kähler manifold. Assume that $M$ has bounded geometry, or that it admits a pluri-subharmonic exhaustion function, then the following statements hold:

(a) If $\pi_{0}^{\infty}(M) \geq 2$ then $b_{1}(M)>0$;

(b) If $\pi_{0}^{\infty}(M) \geq 3$ then there exists a complete Riemann surface $\Sigma$ and a proper, surjective, holomorphic map $h: M \rightarrow \Sigma$ with compact fibers.

Theorem 8.2 (Li-Ramachandran [66]). Let $M$ be a complete Kähler manifold. Suppose $R(x)$ is function defined on $M$ which is a lower bound of the Ricci curvature satisfying

$$
\operatorname{Ric}_{M}(x) \geq R(x)
$$


for all $x \in M$. Let

$$
R_{-}(x)=\max \{-R(x), 0\}
$$

be the negative part of the function $R$ which is assumed to be integrable, i.e.,

$$
\int_{M} R_{-}<\infty
$$

Also assume that

$$
R(x) \geq-\epsilon \rho^{-2}(x),
$$

for some sufficiently small $\epsilon>0$. Then the following statements hold:

(a) If $\pi_{0}^{\infty}(M) \geq 2$ then $M$ must be parabolic and $b_{1}(M)>0$;

(b) If $\pi_{0}^{\infty}(M) \geq 3$ then there exists a complete parabolic Riemann surface $\Sigma$ and a proper, surjective, holomorphic map $h: M \rightarrow \Sigma$ with compact fibers.

Let us remark that the curvature assumption in Theorem 8.2 is sharp. In fact, let us consider $M=\mathbb{C}^{2} \backslash\left\{p_{1}, \ldots, p_{k}\right\}$, where $\left\{p_{i}\right\}$ are $k$ wellspaced points in $\mathbb{C}^{2}$. For any $\delta>0$, there exists [66] a complete Kähler metric on $M$ such that the Ricci curvature satisfies

$$
\operatorname{Ric}_{M}(x) \geq-(1+\delta) \rho^{-2}(x) .
$$

Obviously the conclusion of Theorem 8.2 is invalid. In particular this indicates that $\epsilon$ has to be less than 1 in the assumption of Theorem 8.2. In this example, we can also take the number of points $k$ to be infinite.

Theorem 8.3 (Li [61], [62]). Let $M$ be a complete Kähler manifold with non-negative sectional curvature near infinity. Then the conclusion of Theorem 8.2 holds. Moreover, if $\pi_{o}^{\infty}(M) \geq 3$, then for each end $E$ of $M$, the fibration

$$
h: E \rightarrow h(E) \subset \Sigma
$$

is a Riemannian fibration with fiber given by a compact Kähler manifold, $N$, with non-negative sectional curvature. Locally $E$ is a Riemannian product of $N$ and open subsets $U \subset \Sigma$. Also, $\Sigma$ is a parabolic surface with non-negative curvature near infinity.

Corollary 8.4. If $M$ be a complete Kähler manifold with positive sectional curvature near infinity, then $M$ has most 2 ends.

Using a vanishing theorem of Li-Yau [80], one can prove a rather general theorem which put a restriction on the number of non-parabolic ends for a Kähler manifold. 
Theorem 8.5 (Li-Tam [72]). Let $M$ be a complete Kähler manifold of complex dimension $m$. Suppose $R(x)$ is function defined on $M$ which is a lower bound of the Ricci curvature satisfying

$$
\operatorname{Ric}_{M}(x) \geq R(x)
$$

for all $x \in M$. Let

$$
R_{-}(x)=\max \{-R(x), 0\}
$$

be the negative part of the function $R$. If

$$
\int_{M} R_{-}<\infty
$$

and the $L^{q}$-norm of $R_{-}$over the geodesic ball of radius $r$ centered at some fixed point $p \in M$ satisfies

$$
\int_{B_{p}(r)} R_{-}^{q}=o\left(r^{\beta(q-1)}\right)
$$

for some $q>m$ and $\beta<2 /(m-2)$, then

$$
\Pi_{0}^{\infty}(M) \leq 1
$$

\section{Harmonic functions of polynomial growth}

In 1980, Cheng [24] observed that the localized version of the Yau's gradient estimate (Theorem 5.1) can be used to show that a manifold with non-negative Ricci curvature does not admit any non-constant sublinear growth harmonic functions.

Theorem 9.1 (Cheng [24]). Let $M$ be a complete manifold with nonnegative Ricci curvature. There are no non-constant harmonic functions defined on $M$ which is of sublinear growth, i.e.,

$$
|f(x)| \leq o(\rho(x))
$$

as $x \rightarrow \infty$, where $\rho(x)$ denotes the distance function to some fixed point $p \in M$.

In fact, in the same paper, Cheng proved that a similar statement is true for harmonic maps into a Cartan-Hadamard manifold. Note that on the $n$-dimensional Euclidean space, $\mathbb{R}^{n}$, the set of harmonic polynomials 
generate all the polynomial growth harmonic functions. In particular, for each $d \in \mathbb{Z}^{+}$, the space of harmonic polynomials $\mathcal{H}_{d}\left(\mathbb{R}^{n}\right)$ of degree at most $d$ is of dimension

$$
\begin{aligned}
\operatorname{dim} \mathcal{H}_{d}\left(\mathbb{R}^{n}\right) & =\left(\begin{array}{c}
n+d-1 \\
d
\end{array}\right)+\left(\begin{array}{c}
n+d-2 \\
d-1
\end{array}\right) \\
& \sim \frac{2}{(n-1) !} d^{n-1} .
\end{aligned}
$$

Cheng's theorem asserts that manifolds with non-negative Ricci curvature is quite similar to $\mathbb{R}^{n}$ for harmonic functions which grow sublinearly. In view of this result, and the fact that all polynomial growth harmonic functions in $\mathbb{R}^{n}$ are generated by harmonic polynomials, Yau conjectured that the space of harmonic functions on a manifold with non-negative Ricci curvature of at most polynomial growth at a fixed degree must be of finite dimensional. To state this more precisely, let us define the following spaces of harmonic functions.

Definition 9.2. Let $\mathcal{H}_{d}(M)$ be the space of harmonic functions $f$ defined on a complete manifold $M$ satisfying the growth condition

$$
|f(x)|=O\left(\rho^{d}(x)\right) .
$$

Note that in this notation, $\mathcal{H}_{0}(M)=\mathcal{H}^{\infty}(M)$.

Conjecture 9.3 (Yau [109]). Let $M$ be a complete manifold with non-negative Ricci curvature. The dimension of $\mathcal{H}_{d}(M)$ is finite for all $d \in \mathbb{R}^{+}$.

In fact, Yau also raised the question if

$$
\operatorname{dim} \mathcal{H}_{d}(M) \leq \operatorname{dim} \mathcal{H}_{d}\left(\mathbb{R}^{n}\right)
$$

for manifolds with non-negative Ricci curvature. In 1989, the author and L. F. Tam [70] considered the case when $d=1$.

Theorem 9.4 (Li-Tam [70]). Let $M$ be a complete manifold with non-negative Ricci curvature. Suppose the volume growth of $M$ satisfies

$$
V_{p}(r)=O\left(r^{k}\right)
$$

for some constant $k>0$. Then

$$
\operatorname{dim} \mathcal{H}_{1}(M) \leq \operatorname{dim} \mathcal{H}_{1}\left(\mathbb{R}^{k}\right)=k+1 .
$$


Observe that the assumption on the Ricci curvature and the Bishop comparison theorem assert that

$$
V_{p}(r) \leq \omega_{n} r^{n}
$$

where $\omega_{n}$ is the volume of the unit ball in $\mathbb{R}^{n}$. On the other hand, a theorem of Yau [108] (also see [23]) asserts that $V_{p}(r)$ must grow at least linearly. Hence the constant $k$ in Theorem 9.3 must exist and satisfy $1 \leq k \leq n$.

Corollary 9.5. Let $M$ be a complete manifold with non-negative Ricci curvature. Then

$$
\operatorname{dim} \mathcal{H}_{1}(M) \leq n+1
$$

This theorem leads us to consider two obvious questions.

Question 9.6 (Li-Tam [70]). Let $M$ be a complete manifold with non-negative Ricci curvature. Suppose the volume growth of $M$ satisfies

$$
V_{p}(r)=O\left(r^{k}\right)
$$

for some constant $k>0$. Is it true that

$$
\operatorname{dim} \mathcal{H}_{d}(M) \leq \operatorname{dim} \mathcal{H}_{d}\left(\mathbb{R}^{k}\right)=\left(\begin{array}{c}
k+d-1 \\
d
\end{array}\right)+\left(\begin{array}{c}
k+d-2 \\
d-1
\end{array}\right) ?
$$

The answer to this question was affirmatively verified by Kasue [57] and Li-Tam [71], independently, for the case when $M$ is of dimension 2. In fact, they considered surfaces satisfying a much weaker curvature condition. We will defer the discussion of this until the next section.

Question 9.7. What can we say about the manifold on which equality is achieved in the upper bound given by Corollary 9.5 , or even Theorem 9.4?

The first result in this direction, was due to the author, where he assumed, in addition to non-negative Ricci curvature, that the manifold is Kähler.

Theorem 9.8 (Li [64]). Let $M$ be a complete Kähler manifold with non-negative Ricci curvature. If

$$
\operatorname{dim} \mathcal{H}_{1}(M)=2 m+1
$$


where $m=\operatorname{dim}_{\mathbb{C}}(M)$, then $M$ must be isometrically biholomorphic to $C^{m}$.

Later, Cheeger-Colding-Minicozzi proved this theorem without the Kähler assumption. In fact, they proved a splitting type theorem for the tangent cone at infinity.

Theorem 9.8 (Cheeger-Colding-Minicozzi [20]). Let $M$ be a complete manifold with non-negative Ricci curvature. If

$$
\operatorname{dim} \mathcal{H}_{1}(M)=k+1
$$

then any tangent cone $C(M)$ at infinity of $M$ must spit into $\mathbb{R}^{k} \times N$ where $N$ is a (possibly singular) metric cone. In particular, if

$$
\operatorname{dim} \mathcal{H}_{1}(M)=n+1
$$

then $M$ must be isometric to $\mathbb{R}^{n}$.

In a recent paper, Wang [104] estimated $\operatorname{dim} \mathcal{H}_{1}(M)$ for manifolds with non-negative Ricci curvature outside a compact set and have finite first Betti number.

Theorem 9.10 (Wang [104]). Let $M$ be a complete manifold with non-negative Ricci curvature outside the geodesic ball $B_{p}(a)$ centered at $p \in M$ of radius $a>0$. Assume that the first Betti number of $M$ is finite. Suppose that the Ricci curvature on $B_{p}(a)$ has a lower bound given by

$$
\operatorname{Ric}_{M} \geq-K
$$

for some constant $K>0$. There exist a constant $C(n, a, K)>0$ depending only on $n, a$, and $K$ such that

$$
\operatorname{dim} \mathcal{H}_{1}(M) \leq C(n, a, K) .
$$

\section{Surfaces of finite total curvature}

Definition 10.1. A complete surface $M$ is said to have finite total curvature if the negative part of its Gaussian curvature is integrable. More precisely, if $K(x)$ denotes the Gaussian curvature on $M$ and its negative part is defined by

$$
K_{-}(x)=\max \{-K(x), 0\},
$$


then $M$ has finite total curvature if

$$
\int_{M} K_{-}<\infty .
$$

These kind of surfaces were first studied by Cohn-Vossen [28] in connection to generalizing a Gaussian-Bonnet formula for complete surfaces. He showed that if for any compact exhaustion $\Omega_{i}$ of a complete surface $M$, the sequence

$$
\int_{\Omega_{i}} K \rightarrow \int_{M} K
$$

converges to a possibly infinite limit denoted by $\int_{M} K$, then the inequality

$$
\int_{M} K \leq 2 \pi \chi(M)
$$

holds, where $\chi(M)$ is the Euler characteristic of $M$. This inequality is referred to as the Cohn-Vossen inequality. Later, Huber [53] showed that if

$$
\int_{M} K_{-}<\infty
$$

then $M$ must be conformally equivalent to a compact Riemann surface with finite punctures. Moreover, the Cohn-Vossen inequality is valid. Note that since

$$
K=K_{+}-K_{-}
$$

for

$$
K_{+}(x)=\max \{K(x), 0\}
$$

being the positive part of $K$, the Cohn-Vossen inequality implies that

$$
\int_{M} K_{+} \leq \int_{M} K_{-}+2 \pi \chi(M) .
$$

On the other hand, Huber's theorem asserts that the right hand side is finite, hence

$$
\int_{M} K_{+}<\infty
$$

follows as a consequence. An upshot of this is that

$$
\int_{M} K_{-}<\infty
$$


implies

$$
\int_{M}|K|<\infty .
$$

This justifies the term total curvature in Definition 10.1. After Huber, there were much work done [38], [48], [39] in understanding this class of surfaces. In particular, Hartman - though had not explicitly stated in his paper - showed that the correction term in Cohn-Vossen inequality can be computed in terms of the volume growth of each end. Specifically, since $M$ is conformally equivalent to a compact Riemann surface with finite punctures, $M$ has finitely many ends given by $\left\{e_{i}\right\}_{i=1}^{k}$. Moreover, each end $e_{i}$ is conformally equivalent to a punctured disk, hence must be parabolic. The finite total curvature assumption implies that the volume growth of $M$ is at most quadratic. For each end $e_{i}$, we can define

$$
\alpha_{i}=1-\lim _{r \rightarrow \infty} \frac{V_{e_{i}}(r)}{\pi r^{2}},
$$

with $\alpha_{i} \leq 1$. Hartman showed that

$$
2 \pi \chi(M)-\int_{M} K=2 \pi \sum_{i=1}^{k} \lim _{r \rightarrow \infty}\left(1-\alpha_{i}\right) .
$$

The next theorem indicates that these constants $\alpha_{i}$ also play an important role in the function theory of $M$.

Theorem 10.2 (Li-Tam [71]). Let $M$ be a complete surface with finite total curvature. Then

$$
\operatorname{dim} \mathcal{H}_{d}(M) \leq \sum_{i=1}^{k} \operatorname{dim} \mathcal{H}_{d\left(1-\alpha_{i}\right)}\left(\mathbb{R}^{2}\right),
$$

and if $M$ has quadratic area growth, i.e., $\alpha_{i}<1$ for some $i$, then for any $\epsilon>0$

$$
\operatorname{dim} \mathcal{H}_{d}(M) \geq \sum_{i=1}^{k} \operatorname{dim} \mathcal{H}_{d\left(1-\alpha_{i}\right)-\epsilon}\left(\mathbb{R}^{2}\right)-k^{\prime}
$$

where $k^{\prime}$ is the number of ends with $\alpha_{i}=1$. Here we are taking the convention that

$$
\operatorname{dim} \mathcal{H}_{d}\left(\mathbb{R}^{2}\right)=0
$$

for $d<0$.

This estimate can be sharpen, when the manifold has non-negative curvature near infinity. 
Theorem 10.3 (Li-Tam [71]). Let $M$ be a complete manifold with non-negative Gaussian curvature near infinity, then

$$
\operatorname{dim} \mathcal{H}_{d}(M)=\sum_{\imath=1}^{k} \operatorname{dim} \mathcal{H}_{d\left(1-\alpha_{i}\right)}\left(\mathbb{R}^{2}\right)
$$

for all $d \geq 1$.

Note that when we restrict ourselves to manifolds with non-negative Gaussian curvature, then either $M$ is a cylinder $\mathbb{S}^{1} \times \mathbb{R}$ or $M$ has only one end. In the first case when $M=\mathbb{S}^{1} \times \mathbb{R}$, the polynomial growth harmonic functions on $M$ are generated by the constant function and the linear function $t$ which parameterizes $\mathbb{R}$. Hence

$$
\operatorname{dim} \mathcal{H}_{d}(M)=\left\{\begin{array}{lll}
1 & \text { if } & d<1 \\
2 & \text { if } & d \geq 1
\end{array}\right.
$$

When $M$ has only one end, then according to Theorem 10.3,

$$
\operatorname{dim} \mathcal{H}_{d}(M)=\operatorname{dim} \mathcal{H}_{d(1-\alpha)}\left(\mathbb{R}^{2}\right)
$$

where

$$
1-\alpha=\lim _{r \rightarrow \infty} \frac{V(r)}{\pi r^{2}} .
$$

Hence if $M$ has linear volume growth, then

$$
\operatorname{dim} \mathcal{H}_{d}(M)=1
$$

for all $d \geq 1$. On the other hand, if $M$ has quadratic volume growth, then the curvature assumption implies that $\alpha \geq 0$ and

$$
\operatorname{dim} \mathcal{H}_{d}(M) \leq \operatorname{dim} \mathcal{H}_{d}\left(\mathbb{R}^{2}\right) .
$$

In either case, Question 9.6 is answered affirmatively for surfaces. We should point out that Kasue [57] independently proved the upper bound in Theorem 10.2.

\section{High dimensions}

Before we discuss the higher dimensional development of Yau's conjecture, we would like to point out different points of view of this type of problems. The first is to consider polynomial growth solutions for elliptic operators in $\mathbb{R}^{n}$. Let

$$
L=\frac{\partial}{\partial x^{i}}\left(a_{\imath \jmath} \frac{\partial}{\partial x^{\jmath}}\right)
$$


be an elliptic operator defined on $\mathbb{R}^{n}$ with measurable coefficients $\left(a_{\imath \jmath}\right)$ satisfying the uniformly bounded conditions,

$$
\lambda\left(\delta_{i j}\right) \leq\left(a_{i j}\right) \leq \Lambda\left(\delta_{i j}\right)
$$

for some constants $\lambda, \Lambda>0$. The Harnack inequality of De Giorgi-NashMoser implies that $L$ has no non-constant bounded solutions. In fact, if we define

$$
\mathcal{H}_{d}(L)=\left\{f \in H^{1,2}\left(\mathbb{R}^{n}\right)|L(f)=0,| f \mid(x)=O\left(\rho^{d}\right)\right\}
$$

then the De Giorgi-Nash-Moser theory implies that there exists $d_{0}>0$ depending on $\Lambda / \lambda$, such that,

$$
\operatorname{dim} \mathcal{H}_{d}(L)=1
$$

for all $d<d_{0}$. For general $d$, Avelleneda and Lin [6] first considered the special case when the coefficients $\left(a_{i j}\right)$ are periodic, Lipschitz continuous functions in all the variables. They showed that there is a linear isomorphism between polynomial growth solutions of

$$
L(f)=0
$$

to harmonic polynomials in $\mathbb{R}^{n}$, hence gave a precise estimate on $\operatorname{dim} \mathcal{H}_{d}(L)$. The Lipschitz condition was later dropped in a paper of Moser-Struwe [88]. In a recent work of Lin [81], he considered elliptic operators satisfying both (11.1) and (11.2) plus an asymptotically conic condition (see Definition 2.1 of [81]). The condition roughly says that the operator is asymptotic to a unique conic operator. With this extra condition, Lin proved that

$$
\operatorname{dim} \mathcal{H}_{d}(M)<\infty
$$

for all $d>0$. Moreover, the dimension of each $\mathcal{H}_{d}(M)$ can be estimated explicitly using information on $L$.

Recently, Zhang [110] proved a similar dimension estimate for $\operatorname{dim} \mathcal{H}_{d}(L)$ for a class of uniformly elliptic operators of divergence form that is more general than those in [81]. He considered those operators which are not necessarily asymptotic to a unique conic operator, but those who are asymptotic to a periodic family of conic operators. In this case, he proved that

$$
\operatorname{dim} \mathcal{H}_{d}(L) \leq C d^{n-1} .
$$


Another class of elliptic operators which have some baring to this problem are uniformly elliptic operators of non-divergence form. Let

$$
L=a_{i j} \frac{\partial}{\partial x^{i}} \frac{\partial}{\partial x^{j}}
$$

be an elliptic operator defined on $\mathbb{R}^{n}$ with coefficients $\left(a_{i j}\right)$ satisfying (11.2). Then the Harnack inequality of Krylov-Safonov (see [41]) implies that there exists $d_{0}>0$ depending on $\Lambda / \lambda$, such that,

$$
\operatorname{dim} \mathcal{H}_{d}(L)=1
$$

In yet another direction, Bombieri-Giusti [13] proved a Harnack inequality for uniformly elliptic operators on area minimizing hypersurfaces $M$ in $\mathbb{R}^{n}$. Hence in the same spirit as above,

$$
\operatorname{dim} \mathcal{H}_{d}(M, L)=1
$$

for $d$ sufficiently small, where $L$ is a uniformly elliptic operator on $M$

Recently there has been substantial developments on Yau's conjecture in higher dimensions. We will take this opportunity to document various contributions and give the historical account in this direction. The first partial result was indirectly given by Bando-Kasue-Nakajima [10]. They proved that if the sectional curvature, $K_{M}$ of a complete $n$-dimensional manifold satisfies

$$
\left|K_{M}(x)\right| \leq C \rho^{-(2+\epsilon)}(x)
$$

for some constants $C, \epsilon>0$ and if the volume growth for each end $E$ satisfies

$$
V_{E}(r) \geq C r^{n}
$$

then $M$ is asymptotically locally Euclidean. This fact is sufficient [11], [57], [58] to imply that

$$
\operatorname{dim} \mathcal{H}_{d}(M)<\infty
$$

for all $d$. In a series of papers, Colding-Minicozzi [29], [34] proved a number of theorems which eventually lead to and went beyond Yau's conjecture. First, they proved the case when $M$ has non-negative Ricci curvature and has maximal volume growth. Eventually, they improved their argument to give a dimension estimate for $\operatorname{dim} \mathcal{H}_{d}(M)$ for manifolds satisfying the volume doubling property (7.1) and the Poincaré inequality. 
In the context of this section, we will say that a manifold satisfies the Poincare inequality if there exists a constant $\alpha>0$, such that, the first Neumann eigenvalue for the Laplacian on $B_{x}(r)$ satisfies

$$
\lambda_{1}\left(B_{x}(r)\right) \geq \alpha r^{-2}
$$

for all $x \in M$ and $r>0$. It is worth pointing out that though (11.4) is stronger than the weak Poincare inequality (7.2), a covering argument of Jerison [54] asserts that the volume doubling property (7.1) together with the weak Poincaré inequality (7.2), in fact, imply that Poincaré inequality. In [34], they also considered a volume growth property, which asserts that there exists a constant $\nu>0$ such that

$$
\left(\frac{r^{\prime}}{r}\right)^{\nu} V_{x}(r) \geq V_{x}\left(r^{\prime}\right)
$$

for all $x \in M$ and $0<r<r^{\prime}$. Using these conditions, the main result which they proved can be stated as follows:

Theorem 11.1 (Colding-Minicozzi [32], [34]). Let $M$ be a complete manifold satisfying the Poincaré inequality (11.4).

1) Suppose $M$ also satisfies the volume doubling property (7.1), then there exists a constant $C>0$ depending only on $n$ and $\alpha$, such that,

$$
\operatorname{dim} \mathcal{H}_{d}(M) \leq C d^{\eta}
$$

for all $d>1$.

2) Suppose $M$ also satisfies the volume growth property (11.5), then there exists a constant $C>0$ depending only on $n$ an $\alpha$, such that,

$$
\operatorname{dim} \mathcal{H}_{d}(M) \leq C d^{\nu-1} .
$$

for all $d>1$.

In particular, this confirms Yau's conjecture since manifolds with non-negative Ricci curvature satisfy both the Poincare inequality [16] and the volume growth property [12]. In this case, $\nu=n$. This gives a sharp growth rate as $d \rightarrow \infty$, as indicated by the case when $M=\mathbb{R}^{n}$. We would also like to point out that the first estimate of Colding-Minicozzi using the volume doubling property is not sharp in the power of $d$. The sharp power should be $\eta-1$, since $\eta=n$ if $M=\mathbb{R}^{n}$. Also, the volume doubling property and the volume growth property are related. It is clear that, the volume growth property implies the volume doubling property 
with $\eta=\nu$. Moreover, one can easily argue that the volume doubling property (7.1) implies

$$
\left(\frac{2 r^{\prime}}{r}\right)^{\eta} V_{p}(r) \geq V_{p}\left(r^{\prime}\right)
$$

for $r^{\prime} \geq r$. In this sense, the volume doubling property is weaker than the volume growth property. In view of the relationship between the volume doubling property and the volume growth property, it is convenient to define the weak volume growth property which encapsulate both properties. A manifold is said to have the weak volume growth property if there exists constants $C_{1}>0$ and $\eta>0$ such that

$$
C_{1} \frac{\left(r^{\prime}\right)^{\eta}-r^{\eta}}{r^{\eta}} V_{p}(r) \geq V_{p}\left(r^{\prime}\right)-V_{p}(r)
$$

for all $p \in M$ and $0<r \leq r^{\prime}$.

Due to the work of Grigor'yan [47] and Saloff-Coste [93], [94] it is known that the volume doubling property (7.1) together with the Poincaré inequality (11.4) is equivalent to the parabolic Harnack inequality which implies the elliptic Harnack inequality. Grigor'yan has informed me, by way of an example, that the parabolic Harnack inequality is stronger than the elliptic Harnack inequality. In any case, (7.1) and (11.4) imply a mean value inequality of the form

$$
V_{x}(r) f(x) \leq C_{2} \int_{B_{x}(r)} f
$$

for some constant $C_{2}$, and for any non-negative function $f$ defined on $B_{x}(r)$ satisfying

$$
\Delta f \geq 0 \text {. }
$$

In this case, $C_{2}$ will depend only on $\eta, \alpha$, and $n$. Indeed, it was argued in [94] that (7.1) and (11.4) imply a Sobolev inequality of the form

$$
\begin{aligned}
& \left(\int_{B_{x}(r)} f^{\frac{2 \mu}{\mu-2}}\right)^{\frac{\mu-2}{\mu}} \\
& \quad \leq C_{3} V_{x}(r)^{-2 / \mu}\left(r^{2} \int_{B_{x}(r)}|\nabla f|^{2}+\int_{B_{x}(r)} f^{2}\right)
\end{aligned}
$$

for any compactly supported function $f \in H_{1,2}^{c}\left(B_{x}(r)\right)$, where $C_{3}>0$ and $\mu>2$ are some fixed constants, and $x \in M$ and $r>0$ are arbitrary. It is now clear that by running the Moser iteration argument [87] using 
(7.1) and (11.8), one obtains (11.7). In fact, Moser's argument actually implies the mean value inequality not only for non-negative subharmonic functions, but for non-negative functions satisfying

$$
\Delta f \geq-g f
$$

where $g$ is a non-negative function satisfying some appropriate decay condition (see [63]). In this case, $C_{2}$ in (11.7) will depend on $\mu, C_{3}$ and $g$. In particular, a special case of this situation is when $g$ has compact support.

Colding-Minicozzi circulated an announcement [32] of Theorem 11.1 in June 1996 together with a number of applications using Theorem 11.1. In [33] and [34], they proved many of the announced theorems, including Theorem 11.1. Shortly after the circulation of [34], the author [65] came up with a simple argument using a weaker assumption.

Theorem 11.2 ( $\mathrm{Li}$ [65]). Let $M$ be a complete manifold satisfying the weak volume growth property (11.6). Let $K$ be a linear space of sections of a rank-q vector bundle $E$ over $M$. Suppose each $u \in K$ satisfies the growth condition

$$
|u|(x)=O\left(\rho^{d}(x)\right)
$$

as the distance $\rho$ to some fixed point $p \in M$ goes to infinity for some constant $d \geq 1$, and the mean value inequality

$$
C_{2} \int_{B_{x}(r)}|u|^{2} \geq V_{x}(r)|u|^{2}(x)
$$

for all $x \in M$ and $r>0$. Then there exists a constant $C>0$ depending only on $\eta$ and $C_{1}$, such that

$$
\operatorname{dim} K \leq q C C_{2} d^{\eta-1} .
$$

In their announcement [32], the authors also announced, without indication of the proof, that $\mathcal{H}_{d}(M)$ is finite dimensional if $M$ is a minimal submanifold in Euclidean space with Euclidean volume growth. In the same note, they also announced a finite dimensionality result for polynomial growth harmonic sections of at most degree $d$ on a Hermitian vector bundle with nonnegative curvature over a manifold with non-negative Ricci curvature. Shortly after the circulation of [65], Colding-Minicozzi circulated a new preprint [35] providing the proofs for the minimal submanifold and the harmonic sections cases. In this paper, they also used a 
form of mean value inequality similar to (11.7). However their argument did not provide the sharp power in $d$.

As indicated in the above discussion, as long as $|u|^{2}$ satisfies an inequality of the form (11.9) for some compactly supported $g$, and $M$ also satisfies the Sobolev inequality (11.8), then the mean value inequality follows as a consequence. In particular, the following corollaries can be deduced from Theorem 11.2.

Corollary 11.3. Let $M^{n}$ be a complete manifold satisfying conditions (11.6) and (11.7) for non-negative subharmonic functions. Then

$$
\operatorname{dim} \mathcal{H}_{d}(M) \leq C C_{2} d^{\eta-1}
$$

for all $d \geq 1$. In particular, if $M$ is quasi-isometric to a manifold with non-negative Ricci curvature, then

$$
\operatorname{dim} \mathcal{H}_{d}(M) \leq C d^{n-1}
$$

for all $d \geq 1$.

In view of the discussion after Theorem 7.4, the work of Coulhon and Saloff-Coste [36] together with Theorem 11.2 implies that one can also deal with the case when $M$ is roughly isometric to a manifold with non-negative Ricci curvature.

Corollary 11.4. Let $M^{n}$ be a complete manifold with Ricci curvature bounded from below. Suppose $M$ is isometric at infinity to a manifold with non-negative Ricci curvature, then

$$
\operatorname{dim} \mathcal{H}_{d}(M) \leq C d^{n-1}
$$

for all $d \geq 1$.

Using the fact that the mean value inequality holds for functions satisfying (11.9), Theorem 11.2 also implies the next corollary.

Corollary 11.5. Let $M$ be a manifold whose metric $d s^{2}$ is obtained by a compact perturbation of another metric $d s_{0}^{2}$ which has non-negative Ricci curvature. Suppose

$$
\mathcal{H}_{d}^{p}(M)=\left\{u \in \Lambda^{p}(M)|\delta u=0,| u \mid(x)=O\left(\rho^{d}(x)\right) \text { as } \rho \rightarrow \infty\right\}
$$

denotes the space of harmonic p-forms of at most polynomial growth of degree $d \geq 1$. Then

$$
\operatorname{dim} \mathcal{H}_{d}^{1}(M)=\operatorname{dim} \mathcal{H}_{d}^{n-1}(M) \leq n C d^{n-1} .
$$


If we further assume that $d s_{0}^{2}$ has non-negative curvature operator, then

$$
\operatorname{dim} \mathcal{H}_{d}^{p}(M) \leq\left(\begin{array}{l}
n \\
p
\end{array}\right) C d^{n-1}
$$

Corollary 11.6. Let $M$ be a complete Ricci flat manifold. Suppose $\mathcal{K}_{d}(M)$ is the space of Killing vector fields on $M$ which has polynomial growth of at most degree $d \geq 1$. Then

$$
\operatorname{dim} \mathcal{K}_{d}(M) \leq C d^{n-1}
$$

Corollary 11.7. Let $M^{m}$ be a complete Kähler manifold of complex dimension $m$. Assume that $M$ satisfies conditions (11.6) and (11.7) for non-negative subharmonic functions. Suppose $E$ is a rank-q Hermitian vector bundle over $M$ and that the mean curvature (in the sense defined in [59]) of $E$ is non-positive. Let $\overline{\mathcal{H}}_{d}(M, E)$ be the space of holomorphic sections which is polynomial growth of at most degree $d \geq 1$. Then

$$
\operatorname{dim} \overline{\mathcal{H}}_{d}(M, E) \leq q C \lambda d^{2 m-1}
$$

In particular the space of polynomial growth holomorphic functions of at most degree $d \geq 1$ is bounded by

$$
\operatorname{dim} \overline{\mathcal{H}}_{d}(M) \leq C \lambda d^{2 m-1}
$$

Complex and algebraic geometers have been interested in estimating the dimension of $\overline{\mathcal{H}}_{d}(M, E)$ for many years. We would like to refer to the survey article of Mok [86] for a more detail history and reference in this direction. Another interesting result was due to $\mathrm{Wu}$, Tam and the author, where they considered Kähler manifolds with at most quadratic volume growth. In this case, no additional assumption on the manifold is necessary.

Theorem 11.8 (Wu [105], [106]). Let $M$ be a complete Kähler manifold. If $M$ has subquadratic volume growth

$$
V_{p}(r)=o\left(r^{2}\right)
$$

then $M$ does not admit any non-constant polynomial growth holomorphic functions. If $M$ has quadratic volume growth

$$
V_{p}(r)=O\left(r^{2}\right)
$$


then there exists constants $C(m, d)>0$ depending only on $m$ and $d$ such that

$$
\operatorname{dim} \overline{\mathcal{H}}_{d}(M) \leq C(m, d)
$$

Theorem 11.2 also applies to uniformly elliptic operators on $\mathbb{R}^{n}$. The following corollaries are consequences of the theorem.

Corollary 11.9. Let

$$
L=\frac{\partial}{\partial x^{2}}\left(a_{i j} \frac{\partial}{\partial x^{j}}\right)
$$

be an elliptic operator of divergence form defined on $\mathbb{R}^{n}$ with uniformly bounded coefficients satisfying (11.2). Let

$$
\mathcal{H}_{d}(L)=\left\{u \in H_{1,2}^{\text {loc }}\left(\mathbb{R}^{n}\right)|L(u)=0,| u \mid(x)=O\left(\rho^{d}(x)\right) \text { as } \rho \rightarrow \infty\right\}
$$

be the space of L-harmonic functions that has polynomial growth of degree at most $d \geq 1$. Then

$$
\operatorname{dim} \mathcal{H}_{d}(L) \leq C d^{n-1}
$$

Corollary 11.10. Let

$$
L=a_{\imath j} \frac{\partial^{2}}{\partial x^{i} \partial x^{j}}
$$

be an elliptic operator of non-divergence form defined on $\mathbb{R}^{n}$ with uniformly bounded coefficients satisfying (11.2). Let

$$
\mathcal{H}_{d}(L)=\left\{u \in H_{2, n}^{l o c}\left(\mathbb{R}^{n}\right)|L(u)=0,| u \mid(x)=O\left(\rho^{d}(x)\right) \text { as } \rho \rightarrow \infty\right\}
$$

be the space of $L$-harmonic functions that has polynomial growth of degree at most $d \geq 1$. Then

$$
\operatorname{dim} \mathcal{H}_{d}(L) \leq C d^{n-1}
$$

Corollary 11.11. Let $M^{n}$ be a complete minimal surface in $\mathbb{R}^{N}$. Suppose $\rho_{0}$ is the distance function of $\mathbb{R}^{N}$ with respect to some fixed point $p \in M$. Assume the volume growth of $M$ satisfies

$$
V\left(B_{0}(r) \cap M\right) \leq C r^{n}
$$


where $B_{0}(r) \subset \mathbb{R}^{N}$ is the Euclidean ball center at $p$ of radius $r$. Let $L$ be a uniformly elliptic operator defined on $M$. Suppose $\mathcal{H}_{d}(M, L)$ is the space of $L$-harmonic functions $f$ on $M$ satisfying the growth condition

$$
|f|(x)=O\left(\rho_{0}^{d}(x)\right)
$$

for some $d \geq 1$. Then

$$
\operatorname{dim} \mathcal{H}_{d}(M, L) \leq C d^{n-1}
$$

for some constant $C$ depending on $M$ and the ellipticity constants of $L$.

We would like to comment that, the mean value inequality (11.7) is weaker than the Poincaré inequality (11.5). An interesting fact is that Theorem 11.2 allows one to prove that

$$
\operatorname{dim} \mathcal{H}_{0}(M)<\infty
$$

without implying

$$
\operatorname{dim} \mathcal{H}_{0}(M)=1 \text {. }
$$

On the other hand, Theorem 7.1 asserts that the Poincaré inequality and the volume doubling property imply that

$$
\operatorname{dim} \mathcal{H}_{0}(M)=1 \text {. }
$$

An example of a manifold satisfying the hypothesis of Theorem 11.2 but

$$
\operatorname{dim} \mathcal{H}_{0}(M)>1
$$

is $\mathbb{R}^{n} \sharp \mathbb{R}^{n}$ for $n \geq 3$.

In [35], the authors verified that a complete manifold satisfying the assumptions of Theorem 3.3 has the volume doubling property and the mean value inequality. Hence, Theorem 11.2 applies to this case. In a recent preprint of Tam [101], he relaxed the volume comparison condition of Theorem 3.3. Instead of assuming that the volume comparison condition holds on the whole manifold, he only assumed that it hold for each end individually. Note that since the volume growth of each end may be different, this covers a more general situation. In the same article, he also considered harmonic forms on surfaces of finite total curvature and on manifolds with asymptotically non-negative curvature operator .

Surprisingly, as it turned out, Wang and the author observed that the conditions to ensure the validity of Yau's original conjecture, namely the finite dimensionality of $\mathcal{H}_{d}(M)$, can be weaken. If one does not aim for the sharp order estimate as in Theorem 11.2, the weak volume 
growth property (11.6) can be replaced by a polynomial volume growth assumption. Sometimes it is also convenient to replace the mean value inequality (11.7) by the weak mean value inequality of the form

$$
V_{x}(r) f(x) \leq C_{4} \int_{B_{x}(b r)} f
$$

for some constants $C_{4}>0, b \geq 1$, and for any non-negative subharmonic function $f$ defined on $B_{x}(\beta r)$. Note that if the manifold satisfy the weak volume growth property (11.6), then the weak mean value inequality is equivalent to the mean value inequality. On the other hand, without the weak volume growth property, the weak mean value property is, in general, easier to obtain. For example, if a manifold satisfies the Sobolev inequality (11.8), then the Moser iteration argument yields the weak mean value inequality.

Theorem 11.12 (Li-Wang [76]). Let $M$ be a complete manifold whose volume growth satisfies

$$
V_{x}(r)=O\left(r^{\nu}\right)
$$

as $r \rightarrow \infty$ for some $x \in M$ and $\nu>0$. Assume that $M$ also satisfies the weak mean value inequality (11.10). Then

$$
\operatorname{dim} \mathcal{H}_{d}(M) \leq C_{4}(2 b+1)^{(2 d+\nu)} .
$$

As we pointed out, the Sobolev inequality (11.8) implies the weak mean value inequality. If we choose $f \in H_{1,2}^{c}\left(B_{x}(r)\right)$ to be the nonnegative function satisfying $f=1$ on $B_{x}(1)$, and $f=0$ on $M \backslash B_{x}(2)$, then after applying to (11.8), we conclude that $V_{x}(r)=O\left(r^{\mu}\right)$. Hence, Theorem 11.12 can be stated with only the assumption of (11.8). However, as indicated by a recent paper of Li-Wang [77], one can actually do much better on the estimate if we assume (11.8).

Theorem 11.13 (Li-Wang [77]). Let $M$ be a complete manifold satisfying the Sobolev inequality (11.8). Then

$$
\operatorname{dim} \mathcal{H}_{d}(M) \leq C d^{\mu}
$$

for some constant $C>0$.

It is also worthwhile to point out that Theorem 11.12 can be applied to harmonic sections of vector bundles. In particular, a weaker estimate 
as in Corollary 11.7 holds for manifolds satisfying the conditions of Theorem 11.12. The argument of [65] and [76] also can be applied to study $d$-massive sets [75], which yields interesting applications to the image structure of harmonic maps.

Recently, Sung-Tam-Wang [100] considered the effect of $\operatorname{dim} \mathcal{H}_{d}(M)$ under connected sums. They proved a formula for $\operatorname{dim} \mathcal{H}_{d}\left(M_{1} \sharp M_{2}\right)$ in terms of $\operatorname{dim} \mathcal{H}_{d}\left(M_{1}\right)$ and $\operatorname{dim} \mathcal{H}_{d}\left(M_{2}\right)$.

In an attempt to give an affirmative answer to Question 9.6, Li-Wang [77] recently proved a sharp asymptotic estimate for $\operatorname{dim} \mathcal{H}_{d}(M)$ when $M$ has non-negative sectional curvature.

Theorem 11.14 (Li-Wang [77]). Let $M^{n}$ be a complete manifold with non-negative sectional curvature. Let us define $\alpha$ by

$$
\alpha=\lim _{r \rightarrow \infty} r^{-n} V_{p}(r)
$$

The Bishop comparison theorem asserts that $0 \leq \alpha \leq$ omega $_{n}$ where $\omega_{n}$ is the volume of the unit Euclidean ball. Then the truncated sum of $\operatorname{dim} \mathcal{H}_{d}(M)$ must satisfy

$$
\limsup _{d \rightarrow \text { infty }} d^{-n} \sum_{i=1}^{d} \operatorname{dim} \mathcal{H}_{i}(M) \leq \frac{2 \alpha}{n ! \omega_{n}} .
$$

Moreover, the equality

$$
\limsup _{d \rightarrow \text { infty }} d^{-n} \sum_{i=1}^{d} \operatorname{dim} \mathcal{H}_{i}(M)=\frac{2}{n !}
$$

holds if and only if $M=\mathbb{R}^{n}$.

In another recent paper of Li-Wang [78], they also proved a parallel version of this theorem for uniformly elliptic operators of divergence form. The estimate depends on the ratio of the ellipticity bounds at infinity. In particular, if

$$
L=\frac{\partial}{\partial x^{i}}\left(a_{i j} \frac{\partial}{\partial x^{j}}\right)
$$

is an elliptic operator of divergence form defined on $\mathbb{R}^{n}$ with uniformly bounded measurable coefficients satisfying (11.2). We define the ellipticity bounds $\lambda_{r}$ and $\Lambda_{r}$ on the complement of the Euclidean ball of radius $r$ centered at the origin so that they satisfy

$$
\lambda_{r}\left(\delta_{i j}\right) \leq\left(a_{i j}(x)\right) \leq \Lambda_{r}\left(\delta_{i j}\right)
$$


for all $x \in \mathbb{R}^{n} \backslash B_{0}(r)$. Obviously, both $\lambda_{r}$ and $\Lambda_{r}$ are bounded, monotonic functions, hence we can define

$$
\lambda_{\infty}=\lim _{r \rightarrow \infty} \lambda_{r}
$$

and

$$
\Lambda_{\infty}=\lim _{r \rightarrow \infty} \Lambda_{r} .
$$

Theorem 11.15 (Li-Wang [78]). Let $L$ be a uniformly elliptic operator of divergence form defined on $\mathbb{R}^{n}$. Let

$$
\mathcal{H}_{d}(L)=\left\{u \in H_{1,2}^{\text {loc }}\left(\mathbb{R}^{n}\right)|L(u)=0,| u \mid(x)=O\left(\rho^{d}(x)\right) \text { as } \rho \rightarrow \infty\right\}
$$

be the space of $L$-harmonic functions that has polynomial growth of degree at most $d \geq 1$. Then

$$
\sum_{i=1}^{d} \operatorname{dim} \mathcal{H}_{i}(L) \leq\left(\frac{\Lambda_{\infty}}{\lambda_{\infty}}\right)^{n-1} \frac{2}{n !}(d+2 n)^{n} .
$$

\section{2. $L^{p}$ conditions}

Another type of growth conditions which appear naturally in geometric problems are integrability conditions. For example, a natural question to ask is whether a manifold possesses any non-trivial $L^{2}$ harmonic functions. This was first answered by Yau in 1976. Notice that since the absolute value of a harmonic function is subharmonic, we may generalize this discussion to non-negative subharmonic functions which satisfy some integrability conditions.

Theorem 12.1 (Yau [108]). Let $u$ be a non-negative subharmonic function defined on a complete manifold $M$. If $u \in L^{p}(M)$ for some $p>1$, then $u$ must be identically constant. This constant must be zero if $M$ has infinite volume. In particular, a complete manifold does not admit any non-constant $L^{p}$ harmonic functions for $p>1$.

It turns out that for $p \leq 1$, the situation is not as definitive, but geometrically more interesting. In a joint work of the author and Schoen [67], they studied these cases and found out that the curvature of $M$ plays a role. In fact, the case $p=1$ is also different from the remaining cases $p<1$.

Theorem 12.2 (Li-Schoen [67]). Let $M$ be a complete manifold. Suppose $p \in M$ is a fixed point and $\rho$ is the distance function to $p$. If 
there exists constants $C>0$ and $\alpha>0$ such that the Ricci curvature of $M$ satisfies

$$
\operatorname{Ric}_{M}(x) \geq-C\left(1+\rho^{2}(x)\right)\left(\log \left(1+\rho^{2}(x)\right)^{-\alpha},\right.
$$

then any non-negative $L^{1}$ subharmonic must be identically constant. Moreover, this constant must be zero if $M$ has infinite volume.

Theorem 12.3 (Li-Schoen [67]). Let $M$ be a complete manifold. Suppose $p \in M$ is a fixed point and $\rho$ is the distance function to $p$. There exists a constant $\delta(n)>0$ depending only on $n$, such that, if the Ricci curvature satisfies $M$ satisfies

$$
\operatorname{Ric}_{M}(x) \geq-\delta(n) \rho^{-2}(x)
$$

as $x \rightarrow \infty$, then any non-negative $L^{p}$ subharmonic must be identically constant for $p \in(0,1)$. Moreover, this constant must be zero if $M$ has infinite volume.

In the same paper, $\mathrm{Li}$ and Schoen also produced examples of manifolds which possess non-constant $L^{p}$ harmonic functions. They showed that for any $\epsilon>0$, there are manifolds with sectional curvature decay at the order of

$$
K_{M} \sim-C \rho^{2+\epsilon}
$$

as $\rho \rightarrow \infty$, which admit non-constant $L^{1}$ harmonic functions. Also, for any $p<1$, there exists manifolds with sectional curvature behave like

$$
K_{M} \sim-C \rho^{-2}
$$

as $\rho \rightarrow \infty$ which admit non-constant $L^{p}$ harmonic functions. These examples show that the curvature condition in Theorem 12.3 is sharp and the condition in Theorem 12.2 is almost sharp. In fact, a sharp curvature condition was later found by the author for the case $p=1$.

Theorem 12.4 ( $\mathrm{Li}[60])$. Let $M$ be a complete manifold. Suppose $p \in M$ is a fixed point and $\rho$ is the distance function to $p$. If there exists a constant $C>0$ such that the Ricci curvature of $M$ satisfies

$$
\operatorname{Ric}_{M}(x) \geq-C\left(1+\rho^{2}(x)\right),
$$

then any non-negative $L^{1}$ subharmonic function must be identically constant. Moreover, this constant must be zero if $M$ has infinite volume.

Other than lower bounds on the Ricci curvature, there are also other conditions which will imply the non-existence of $L^{p}$ harmonic functions.

Theorem 12.5 (Li-Schoen [67]). Let $M$ be a complete manifold satisfying one of the following conditions: 
- $M$ is a Cartan-Hadamard manifold.

- $M$ has Ricci curvature bounded from below and the volume of every unit geodesic ball is uniformly bounded from below.

Then for all $p \in(0,1]$, any non-negative $L^{p}$ subharmonic function must be constant.

For the sake of application, Yau's theorem can be relaxed to the following form:

Proposition 12.6 (Yau [108]). Let $M$ be a complete manifold. Suppose $u$ is a non-negative subharmonic function whose $L^{p}$-norm satisfies the growth condition

$$
\int_{B_{x}(r)} u^{p}=o\left(r^{2}\right)
$$

as $r \rightarrow \infty$ for some fixed point $x \in M$. Then $u$ must be identically constant. Moreover, this constant must be zero if the volume growth of $M$ satisfies

$$
\limsup _{r \rightarrow \infty} r^{-2} V_{x}(r)>0
$$

as $r \rightarrow \infty$.

The interested reader should also refer to the work of Nadirashvili [89] for a different type of integrability condition for the Liouville theorem.

\section{Cartan-Hadamard manifolds}

The function theory on a hyperbolic disk is quite different from the Euclidean plane. Our previous discussion, in many ways models on the Euclidean case. In this section, we will discuss the higher dimensional analog of the hyperbolic case. With the intend of proving a uniformization type theorem for higher dimensional Kähler manifolds, Greene-Wu and Yau asked if a complete, simply connected, Kähler manifold with sectional curvature bounded from above by -1 is biholomorphic to a bounded domain in $\mathbb{C}^{n}$. Clearly, to prove such a statement, one needs to produce many bounded holomorphic functions to be used as embedding functions. The first step is to study the real analog of this statement and see if one can produce enough bounded harmonic functions. In fact, Greene-Wu [42] posted the following conjecture:

Conjecture 13.1 (Greene-Wu [42]). Let $M$ be a Cartan-Hadamard manifold whose sectional curvature satisfies the upper bound

$$
K_{M}(x) \leq-C \rho^{-2}(x)
$$


for some constant $C>0$, where $\rho$ is the distance function to a fixed point. Then $M$ must admit a non-constant bounded harmonic function.

In 1983, Sullivan [98] proved that there are abundance of bounded harmonic functions on a strongly negatively curved Cartan-Hadamard manifold. Anderson [4] later used an argument of Choi [27] gave another proof of the same statement. To describe the space of bounded harmonic functions on Cartan-Hadamard manifold with strongly negative curvature, we need to define the geometric boundary.

Definition 13.2. Let $M$ be a Cartan-Hadamard manifold. We define the geometric boundary $M(\infty)$ of $M$ to be the set of equivalent classes of geodesic rays defined by the equivalence relation that two geodesic rays $\gamma_{1}(t)$ and $\gamma_{2}(t)$ are equivalent if $\rho\left(\gamma_{1}(t), \gamma_{2}(t)\right)$ is a bounded function to $t \in[0, \infty)$.

The geometric boundary $M(\infty)$ together with $M$ form a compactification of $M$, and $M \cup M(\infty)$ has a natural topology inherited from $M$, namely the cone topology. The cone $C_{p}(v, \delta)$ about a tangent vector $v \in T_{p} M$ of angle $\delta$ is defined by

$$
C_{p}(v, \delta)=\left\{x \in M \mid \text { the geodesic } \gamma \text { joining } p \text { to } x \text { satisfies }\left\langle\gamma^{\prime}, v\right\rangle<\delta\right\} \text {. }
$$

The open sets of the cone topology is generated by the sets of all truncated cones $C_{p}(v, \delta) \backslash B_{p}(r)$ and geodesic balls $B_{q}(r)$, for $p, q \in M$, $v \in T_{p} M, \delta>0$, and $r>0$. Using the Toponogov comparison theorem, one checks [5] that if the sectional curvature of $M$ is strongly negative, i.e.,

$$
-a \geq K_{M} \geq-b
$$

for some constants $0<a \leq b<\infty$, then $M(\infty)$ has a natural $C^{a / b}$ structure.

Theorem 13.3 (Sullivan [98], Anderson [4]). Let $M$ be a complete, simply connected manifold. Assume that the sectional curvature of $M$ satisfy the bound

$$
-a \geq K_{M} \geq-b
$$

for some constants $0<a \leq b<\infty$. Then for any continuous function $\phi$ defined on the geometric boundary $M(\infty)$ of $M$, there exists a function $f$ defined on $M \cup M(\infty)$ which is harmonic on $M$, and

$$
f=\phi
$$

on $M(\infty)$ 
Shortly after this theorem, Anderson and Schoen considered the existence of positive harmonic functions on the same class of manifolds. In fact, they showed that the Martin boundary is homeomorphic to the geometric boundary. The Martin boundary is defined on non-parabolic manifolds. Let $p \in M$ be a fixed point and $x, y \in M$. Suppose $G$ is the minimal positive Green's function defined on $M$, then we defined the normalized Green's function with pole at $y$ by

$$
h_{y}(x)=\frac{G(y, x)}{G(y, p)} .
$$

Clearly, the normalization yields $h_{y}(p)=1$. Let $y_{i}$ be a non-convergent sequence of points in $M$, then the sequence $\left\{h_{i}(x)=h_{y_{i}}(x)\right\}$ of harmonic functions are uniformly bounded on compact subsets of $M$. The Harnack inequality implies that there exists a subsequence $\left\{h_{i_{j}}\right\}$ which converges uniformly on compact subsets to a positive harmonic function $\bar{h}$ defined on $M$ with the property that $\bar{h}(p)=1$. The corresponding subsequence of points $\left\{y_{i}\right\}$ is denoted to be a fundamental sequence. We say that two fundamental sequences are equivalent if the corresponding limiting harmonic functions are the same.

Definition 13.4. The Martin boundary $\mathcal{M}$ of a manifold $M$ consists of the equivalent classes of fundamental sequences $\bar{y}=\left[y_{i}\right]$. To each $\bar{y} \in \mathcal{M}$ there associates a positive harmonic function $h_{\bar{y}}$ from the above construction.

The Martin boundary together with $M$ form a compactification for $M$. The topology on $M \cup \mathcal{M}$ can be defined by the distance function $\bar{\rho}$ given by

$$
\bar{\rho}\left(y, y^{\prime}\right)=\sup _{x \in B_{p}(1)}\left|h_{y}(x)-h_{y^{\prime}}(x)\right| .
$$

One checks readily that this structure is independent of the choice of $p$, and this topology coincides with the topology induced by the Riemannian structure of $M$.

When $M$ is a complete manifold with non-negative Ricci curvature near infinity and if $M$ has finite first Betti number and it is non-parabolic, then one can show that the Martin boundary consists of $\pi_{0}^{\infty}(M)$ points. The compactification $M \cup \mathcal{M}$ is simply a 1-point compactification at each end of $M$.

Theorem 13.5 (Anderson-Schoen [5]). Let $M$ be a complete, simply connected manifold. Assume that the sectional curvature of $M$ satisfies the bound

$$
-a \geq K_{M} \geq-b
$$


for some constants $0<a \leq b<\infty$. Then the Martin boundary $\mathcal{M}$ is homeomorphic to the geometric boundary and the homeomorphism is $C^{\alpha}$. In particular, there exist a Poisson kernel $K(x, \bar{y})$ defined on $M \times M(\infty)$ which is $C^{\alpha}$ in the variable $\bar{y}$ such that for any positive harmonic function $f$, there exists a unique, finite, positive, Borel measure d $\mu$ defined on $M(\infty)$ such that

$$
f(x)=\int_{M(\infty)} K(x, \bar{y}) d \mu(\bar{y})
$$

Two years later, Ancona [3] generalized this theorem to a larger class of second order elliptic operator. In particular, a special case of Ancona's theorem asserts that the conclusion of Theorem 13.2 holds for manifolds which are quasi-isometric to a strongly negatively curved Cartan-Hadamard manifold.

Theorem 13.6 (Ancona [3]). Let $M$ be a complete, simply connected manifold. Assume that $M$ is quasi-isometric to a manifold $N$ satisfying the curvature bound

$$
-a \geq K_{N} \geq-b
$$

for some constants $0<a \leq b<\infty$. Then the Martin boundary $\mathcal{M}$ of $M$ is homeomorphic to $N_{\infty}$.

Other progress has been made to relax the curvature assumption of these theorems. For example, in [52], Hsu and Marsh relaxed the bounds on the curvature assumption. They generalized Theorem 13.3 to CartanHadamard manifolds whose section curvature satisfies the estimate

$$
-C \rho^{-2} \geq K_{M} \geq-b
$$

for some constants $b>0$ and $C>2$. In 1992, Borbély [14] relaxed the lower bound by assuming that the sectional curvature satisfies

$$
-a \geq K_{M} \geq-b e^{\lambda r}
$$

for some constants $0<a \leq b<\infty$ and $\lambda<1 / 3$. In this case, he proved that the Dirichlet problem at infinity can be solved as in Theorem 13.3. Recently, Cheng proved the existence of non-constant bounded harmonic functions by assuming a pointwise curvature pinching condition.

Theorem 13.7 (Cheng [25]). Let $M$ be a Cartan-Hadamard manifold. Assume that the lower bound of the spectrum $\lambda_{1}(M)$ for the Laplacian on $M$ is positive. Suppose there exists $p \in M$ and a constant $C>0$ such that the sectional curvatures $K_{M}(\sigma)$ and $K_{M}\left(\sigma^{\prime}\right)$ satisfy

$$
\left|K_{M}(\sigma)\right| \leq C\left|K_{M}\left(\sigma^{\prime}\right)\right|
$$


for any pair of 2-plane sections $\sigma$ and $\sigma^{\prime}$ at $x$ containing the tangent vector of the geodesic joining $x$ to $p$. Then for any continuous function $\phi$ defined on the geometric boundary $M(\infty)$ of $M$, there exists a function $f$ defined on $M \cup M(\infty)$ which is harmonic on $M$, and

$$
f=\phi
$$

on $M(\infty)$.

Note that unlike the previous theorems in this section, Cheng's theorem allows points where the curvature of $M$ may vanish. The following theorem of Ballmann also allows this possibility, but rather than a pinching condition he assumed that the manifold is of rank one.

Definition 13.8. A Cartan-Hadamard manifold is said to have rank one if it admits a geodesic $\sigma$ with no parallel Jacobi field along $\sigma$ perpendicular to $\sigma^{\prime}$.

If $M$ is a Cartan-Hadamard manifold which is irreducible and admits a discrete, co-compact, isometry group, then it is known [7], [15] that either $M$ has rank one or $M$ is a symmetric space of noncompact type of rank at least 2 .

Theorem 13.9 (Ballmann [8]). Let $M$ be an irreducible, CartanHadamard manifold which admits a discrete, co-compact isometry group. If $M$ has rank one, then for any continuous function $\phi$ defined on the geometric boundary $M(\infty)$ of $M$, there exists a function $f$ defined on $M \cup M(\infty)$ which is harmonic on $M$, and

$$
f=\phi
$$

on $M(\infty)$.

In a subsequent joint paper of Ballmann and Ledrappier [9], they showed that, in fact, one can represent any bounded harmonic function on $M$ by a Poisson representation formula.

Theorem 13.10 (Ballmann-Ledrappier [9]). Let $M$ be an irreducible, Cartan-Hadamard manifold which admits a discrete, co-compact isometry group. If $M$ has rank one, then there exists an equivalent class of harmonic measures $d \nu_{p}$ defined on $M(\infty)$ for each $p \in M$, such that, for any bounded measurable function $\phi$ the bounded function defined by

$$
f(x)=\int_{M(\infty)} \phi(\bar{x}) d \nu_{x}(\bar{x})
$$


is a harmonic extension of $\phi$ to $M \cup M(\infty)$. Conversely, any bounded harmonic function $f$ can be such represented by some bounded measurable function $\phi$ on $M(\infty)$.

In view of the theorems of Cheng, Ballmann, and Ballmann-Ledrappier, the natural questions to ask is whether it is true that the Martin boundaries of these manifolds are the same as their geometric boundaries? Also, is there a Poisson representation formula similar to the case of strongly negatively curved Cartan-Hadamard manifolds? What can one say about the Martin boundary for manifolds which are quasi-isometric to these manifolds?

Obviously, the set of positive harmonic functions on a complete manifold does not form a vector space. However, if $f$ and $g$ are positive harmonic functions then linear combinations of the form

$$
a f(x)+b g(x)
$$

where $a, b>0$, is again a positive harmonic function. Hence the set of positive harmonic functions form a convex positive cone in a vector space. The boundary points of this convex cone determines the cone itself. A positive harmonic function $f$ which is a boundary point of this cone has the property that if $g$ is another positive harmonic function satisfying

$$
g(x) \leq f(x)
$$

then

$$
g(x)=a f(x)
$$

for some constant $0<a \leq 1$. This property is called minimal. The set of positive harmonic functions are given by the positive span of minimal positive harmonic functions. In his paper [40], Freire considered the Martin boundary for the product of Riemannian manifolds by studying the set of minimal positive harmonic functions.

Theorem 13.11 (Freire [40]). Let $M=M_{1} \times M_{2}$ be a product to two complete Riemannian manifolds whose Ricci curvatures are bounded from below. If $f$ is a minimal positive harmonic function defined on $M$, then $f(x)=f\left(x_{1}, x_{2}\right)$ can be written as a product

$$
f(x)=f_{1}\left(x_{1}\right) f_{2}\left(x_{2}\right)
$$

of positive functions defined on each factor. Moreover, the functions $f_{i}>0$ satisfies

$$
\Delta_{\imath} f_{i}\left(x_{i}\right)=\lambda_{i} f_{i}\left(x_{i}\right)
$$


on $M_{i}$ with constants $\lambda_{i}$ for $i=1,2$ such that $\lambda_{1}+\lambda_{2}=0$. Also, each $f_{i}$ is a minimal positive $\left(\Delta_{i}-\lambda_{i}\right)$-harmonic function. Conversely, the product of two minimal positive $\left(\Delta_{\imath}-\lambda_{i}\right)$-harmonic functions as above yields a minimal positive harmonic function on $M$.

\section{References}

[1] U. Abresch, Lower curvature bounds, Toponogov's theorem, and bounded topology, Ann. Sci. École Norm. Sup. 18 (1985) 651-670.

[2] _ Lower curvature bounds, Toponogov's theorem, and bounded topology. II, Ann. Sci. École Norm. Sup. 20 (1987) 475-502.

[3] A. Ancona, Negatively curved manifolds, elliptic operators, and the Martin boundary, Ann. of Math. 125 (1987) 495-536.

[4] M. Anderson, The Dirichlet problem at infinity for manifolds of negative curvature, J. Differential Geom. 18 (1983) 701-721.

[5] M. Anderson \& R. Schoen, Positive harmonic functions on complete manifolds of negative curvature, Ann. of Math. 121 (1985) 429-461.

[6] M. Avellaneda \& F. H. Lin, Une thèorème de Liouville pour des èquations elliptique à coefficients pèriodiques, Compt. Rendus Acad. Sci. Paris 309 (1989) 245-250.

[7] W. Ballmann, Nonpositively curved manifolds of higher rank, Ann. of Math. 122 (1982) 131-144.

[8] - On the Dirnchlet Problem at infinity for manifolds of nonpositive curvature, Forum Math. 1 (1989) 201-213.

[9] W. Ballmann \& F. Ledrappier, Poisson boundary for rank one manifolds and their cocompact lattices, Forum Math. 6 (1994) 301-313.

[10] S. Bando, A. Kasue \& H. Nakajima, On a construction of coordinates at infinity on manifolds with fast curvature decay and maximal volume growth, Invent. Math. 97 (1989) 313-349.

[11] R. Bartnik, The mass of an asymptotically flat manifold, Comm. Pure Appl. Math. 39 (1986) 661-693.

[12] R. Bishop \& R. Crittenden, Geometry of manifolds, Academic Press, New York and London, 1964.

[13] E. Bombieri \& E. Giusti, Harnack's inequality for elliptic differentral equatıons on minimal surfaces, Invent. Math. 15 (1972) 24-46.

[14] A. Borbély, A note on the Dirichlet problem at infinity for manifolds of negative curvature, Proc. Amer. Math. Soc. 114 (1992) 865-872. 
[15] K. Burns \& R. Spatzier, Manifolds of nonpositvve curvature and their buildings, Inst. Hautes Études Sci. Publ. Math. 65 (1987) 35-59.

[16] P. Buser, On Cheeger's inequality $\lambda_{1} \geq h^{2} / 4$ in Geometry of the Laplace operator, Proc. Symp. Pure Math., Amer. Math. Soc. 36 (1980) 29-77.

[17] M. Cai, Ends of Riemannian manifolds with nonnegative Ricci curvature outside a compact set, Bull. Amer. Math. Soc. 24 (1991) 371-377.

[18] M. Cai, T. Colding \& D. Yang, A gap theorem for ends of complete manifolds, Proc. Amer. Math. Soc. 123 (1995) 247-250.

[19] H. Cao, Y. Shen \& S. Zhu, The structure of stable minimal hypersurfaces in $\mathbb{R}^{n+1}$, Preprint.

[20] J. Cheeger, T. Colding \& W. Minicozzi, Linear growth harmonic functions on complete manifolds with non-negative Ricci curvature, Geom. Func. Anal. 5 (1995) 948-954.

[21] J. Cheeger \& D. Gromoll, The splitting theorem for manifolds of nonnegative Ricci curvature, J. Differential Geom. 6 (1971) 119-128.

[22] - On the structure of complete manifolds of nonnegative curvature, Ann. of Math. 92 (1972) 413-443.

[23] J. Cheeger, M. Gromov \& M. Taylor, Finite propagation speed, kernel estimates for functions of the Laplace operator, and the geometry of complete Riemannian manifolds, J. Differential Geom. 17 (1983) 15-53.

[24] S. Y. Cheng, Liouville theorem for harmonic maps, Geometry of the Laplace operator, Proc. Symp. Pure Math. Amer. Math. Soc. 36 (1980) 147-151.

[25] —_ The Dirichlet problem at infinity for nonpositively curved manifolds, Comm. Anal. Geom. 1 (1993) 101-112.

[26] S. Y. Cheng \& S. T. Yau, Differential equations on Riemannian manifolds and their geometric applications, Comm. Pure Appl. Math. 28 (1975) 333-354.

[27] H. I. Choi, Asymptotic Diruchlet problems for harmonic functions on Riemannian manifolds, Trans. Amer. Math. Soc. 281 (1984) 691-716.

[28] S. Cohn-Vossen, Kürzeste Wege and Totalkrümmung auf Flächen, Compositio Math. 2 (1935) 69-133.

[29] T. Colding \& W. Minicozzi, On function theory on spaces with a lower Ricci curvature bound, , Math. Res. Lett. 3 (1996) 241-246.

[30] - Harmonıc functions with polynomial growth, Preprint.

[31] _ L Large scale behavior of kernels of Schrödinger operators, Preprint.

[32] _ Generalized Liouville properties for manifolds, Preprint.

[33] _ Harmonıc functıons on manifolds, Preprint. 
[34] - Weyl type bounds for harmonıc functıons, Preprint.

[35] - Liouville theorems for harmonic sections and applications manifolds, Preprint.

[36] Th. Coulhon \& L. Saloff-Coste, Variétés ruemanniennes isométrqques à l'infini, Revista Mat. Iber. 11 (1995) 687-726.

[37] H. Donnelly, Bounded harmonic functions and positive Ricci curvature, Math. Z. 191 (1986) 559-565.

[38] F. Fiala, Le problème des isopérimètres sur les surfaces ouvertes à courbure positıve, Comm. Math. Helv. 13 (1940) 293-346.

[39] R. Finn, On a class of conformal metrics with application to differential geometry in the large, Comm. Math. Helv. 40 (1965) 1-30.

[40] A. Freire, On the Martin boundary of Riemannian products, J. Differential Geom. 33 (1991) 215-232.

[41] D. Gilbarg \& N. S. Trudinger, Elliptic partial differential equations of second order, 2nd Ed. Springer, Berlin, 1983.

[42] R. E. Greene \& H. Wu, Function theory on manifolds which possess a pole, Lect. Notes in Math., Springer, Berlin, Vol. 699.

[43] A. Grigor'yan, On the existence of a Green function on a manifold, Uspechi Matem. Nauk 38 (1983) 161-162; Engl transl: Russian Math. Surveys 38 (1983) 190-191.

[44] On the existence of positive fundamental solution of the Laplace equation on Riemannian manifolds, Mat. Sbornik 128 (1985) 354-363; Engl transl: Math. USSR Sbornik 56 (1987) 349-358.

[45] - On Liouville theorems for harmonic functions with finite Dirichlet integral, Math. USSR Sbornik 60 (1988) 485-504.

[46] - On the dimension of spaces of harmonic functions, Math. Notes 48 (1990) 1114-1118.

[47] - The heat equation on noncompact Riemannian manifolds, Math. USSR Sbornik 72 (1992) 47-77.

[48] P. Hartman, Geodesic parallel coordinates in the large, Amer. J. Math. 86 (1964) 705-727.

[49] I. Holopainen, Solutions of elliptic equations on manifolds with roughly Euclidean ends, Math. Z. 217 (1994) 459-477

[50] - Volume growth, Green's functions, and parabolicity of ends, Duke Math. J. 97 (1999) 319-346.

[51] I. Holopainen \& P Koskela, Volume growth and parabolicity, Preprint.

[52] P Hsu \& P Marsh, The limitıng angle of certain Riemannıan Brownıan motions, Comm. Pure Appl. Math. 38 (1985) 755-768. 
[53] A. Huber, On subharmonıc functions and differentıal geometry in the large, Comm. Math. Helv. 32 (1957) 13-72.

[54] D. Jerison, The Poincaré inequality for vector fields satisfyıng Hörmander's condition, Duke Math. J. 53 (1986) 503-523.

[55] M. Kanai, Rough isometries, and combınatorial approximations of geometries of non-compact Riemannıan manifolds, J. Math. Soc. Japan 37 (1985) 391-413.

[56] _ Rough isometries and the parabolicity of Riemannian manifolds, J. Math. Soc. Japan 38 (1986) 227-238.

[57] A. Kasue, Harmonic functions with growth conditions on a manifold of asymptotically non-negative curvature. II, Recent Topics in Differential and Analytic Geometry Adv. Stud. Pure Math., North-Holland, 1989, Vol. 18.

[58] J. L. Kazdan, Parabolicity and the Liouville property on complete Riemannian manifolds, Seminar on New Results in Nonlinear Partial Differential Equations, Max-Planck-Inst. Bonn, 1987, 153-166.

[59] S. Kobayashi, Differential geometry of complex vector bundles, Princeton Univ. Press, Princeton, 1987.

[60] P. Li, Uniqueness of $L^{1}$ solutions for the Laplace equation and the heat equation on Riemannian manifolds, J. Differential Geom. 20 (1984) 447-457.

[61] _ On the structure of complete Kähler manifolds with nonnegative curvature near infinity, Invent. Math. 99 (1990) 579-600.

[62] __ Erratum "On the structure of complete Kähler manifolds with nonnegative curvature near infinity," Invent. Math. 104 (1991) 447-448.

[63] Lecture notes on geometric analysis, Lecture Notes Series No. 6, Research Institute of Mathematics and Global Analysis Research Center, Seoul, Seoul National University, 1993.

[64] Harmonic functıons of linear growth on Kähler manifolds with non-negative Ricci curvature, Math. Res. Lett. 2 (1995) 79-94.

[65] _ Harmonic sections of polynomial growth, Math. Res. Lett. 4 (1997) $35-44$.

[66] P. Li \& M. Ramachandran, Kähler manifolds with almost non-negative Riccı curvature, Amer. J. Math. 118 (1996) 341-353.

[67] P. Li \& R. Schoen, $L^{p}$ and mean value properties of subharmonic functions on Riemannian manifolds, Acta Math. 153 (1984) 279-301.

[68] P. Li \& L. F. Tam, Positive harmonic functions on complete manifolds with nonnegative curvature outside a compact set, Ann. of Math. 125 (1987) 171-207.

[69] Symmetric Green's functions on complete manifolds, Amer. J. Math. 109 (1987) 1129-1154. 
[70] L Linear growth harmonic functions on a complete manifold, J. Differential Geom. 29 (1989) 421-425.

[71] _ Complete surfaces with finite total curvature, J. Differential Geom. 33 (1991) 139-168.

[72] _ Harmonic functions and the structure of complete manifolds, J. Differential Geom. 35 (1992) 359-383.

[73] _ Green's functions, harmonic functions, and volume comparison, J. Differential Geom. 41 (1995) 277-318.

[74] P. Li, L. F. Tam \& J. Wang, Some sharp bounds for the Green's function and the heat kernel, Math. Res. Lett. 4 (1997) 589-602.

[75] P. Li \& J. P. Wang, Convex hull properties of harmonic maps, J. Differential Geom. 48 (1998) 497-530.

[76] _ Mean value inequalities, Indiana Math. J. 48 (1999) 1257-1283.

[77] — Counting massive sets and dimensıons of harmonic functions, J. Differential Geom. 53 (1999) 237-278.

[78] Counting dimensions of L-harmonic functions, Ann. of Math. 152 (2000) 645-658.

[79] P. Li \& S. T. Yau, On the parabolic kernel of the Schrödinger operator, Acta Math. 156 (1986) 153-201.

[80] _ Curvature and holomorphic mappıngs of complete Kähler manifolds, Compositio Math. 73 (1990) 125-144.

[81] F. H. Lin, Asymptotically conic elliptic operators and Liouville type theorems, Volume dedicated to S. Hildebrandt on the occasion of his 60th birthday, (ed. J. Jost), to appear.

[82] Z. Liu, Ball covering property and nonnegative Ricci curvature outside a compact set, Differential Geometry: Riemannian Geometry, Proc. Sypm. Pure Math. Amer. Math. Soc. 54 (3) (1993) 459-464.

[83] T. J. Lyons, Instability of the Liouville property for quast-isometric Riemannian manifolds and reversible Markov chains, J. Differential Geom. 26 (1987) 33-66.

[84] B. Malgrange, Exıstence et approxımatıon des solutions der équations aux dérivées partielles et des équations de convolution, Ann. Inst. Fourier 6 (1955) 271-355.

[85] J. H. Michael \& L. Simon, Sobolev and mean-value inequalities on generalized submanifolds of $\mathbb{R}^{n}$, Comm. Pure Appl. Math. 26 (1973) 361-379.

[86] N. Mok, A survey on complete noncompact Kähler manifolds of positive curvature, Complex analysis of several variables, Proc. Symp. Pure Math. Amer. Math. Soc. 41 (1984) 151-162.

[87] J. Moser, On Harnack's theorem for elliptic differential equations, Comm. Pure Appl. Math. 14 (1961) 577-591. 
[88] J. Moser \& M. Struwe, On a Liouville-type theorem for linear and nonlinear elliptic differential equations on a torus, Bol. Soc. Brasil Mat. 23 (1992) 1-20.

[89] N. Nadirashvili, A theorem of Lıouville type on a Riemannıan manifold, Russian Math. Surveys 40 (1986) 235-236.

[90] M. Nakai, On Evans potentıal, Proc. Japan Acad. 38 (1962) 624-629.

[91] T. Napier \& M. Ramachandran, Structure theorems for complete Kähler manifolds and applications to Lefschetz type theorems, Geom. Funct. Anal. 5 (1995) 809-851.

[92] H. Royden, Harmonıc functions on open Riemann surfaces, Trans. Amer. Math. Soc. 73 (1952) 40-94.

[93] L. Saloff-Coste, Uniformly elliptic operators on Riemannian manifolds, J. Differential Geom. 36 (1992) 417-450.

[94] _ A note on Poincaré, Sobolev, and Harnack inequalities, Internat. Math. Res. Notices 2 (1992) 27-38.

[95] L. Sario, M. Schiffer \& M. Glasner, The span and principal functions in Riemannian spaces, J. Anal. Math. 15 (1965) 115-134.

[96] J. P. Sha \& D. G. Yang, Examples of manifolds of positive Ricci curvature, J. Differential Geom. 29 (1989) 95-103.

[97] R. Schoen \& S. T. Yau, Harmonic maps and the topology of stable hypersurfaces and manifolds of nonnegative Ricci curvature, Comm. Math. Helv. 39 (1976) 333-341.

[98] D. Sullivan, The Dirichlet problem at infinity for a negatively curved manifold, J. Differential Geom. 18 (1983) 723-732.

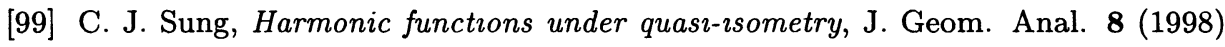
143-161

[100] C. J. Sung, L. F. Tam \& J. P. Wang, Spaces of harmonic functions, J. London Math. Soc., to appear.

[101] L. F. Tam, A note on harmonic forms on complete manifolds, Preprint.

[102] N. Varopoulos, The Porsson kernel on positively curved manifolds, J. Funct. Anal. 44 (1981) 359-380.

[103] _ Potentral theory and diffusion on Riemannian manifolds, Conference on harmonic analysis in honor of Antoni Zygmund, Vol I, II Belmont, Calif., Wadsworth Math. Ser., Wadsworth, 1983, 821-837

[104] J. Wang, Linear growth harmonic functıons on complete manifolds, Comm. Anal. Geom. 4 (1995) 683-698.

[105] H. Wu, Polynomial functions on complete Kähler manifolds, Several complex variables and complex geometry, Proc. Symp Pure Math, Amer. Math. Soc. 52 (1989) 601-610. 
[106] Subharmonic functions and the volume of a noncompact manifold, Differential Geometry, Pitman Monographs Surveys Pure Appl. Math. 52 (1991) 351-368.

[107] S. T. Yau, Harmonıc functions on complete Riemannıan manifolds, Comm. Pure Appl. Math. 28 (1975) 201-228.

[108] - Some function-theoretic properties of complete Riemannian manifolds and their applications to geometry, Indiana Math. J. 25 (1976) 659-670.

[109] - Nonlinear analysis in geometry, L'Enseignement Mathématique, Série des Conférences de l'Union Mathématique Internationale, No. 8, Genève, SROKUNDIG, 1986.

[110] L. Zhang, On generic eigenvalue flow of a family of metrics and its application in dimension estimates of polynomial growth harmonic functions, Comm. Anal. Geom. 7 (1999) 259-278.

UNIVERSity of CALIForNia, IRVINe 